NBER WORKING PAPER SERIES

\title{
DYNAMICAL STRUCTURE AND SPECTRAL PROPERTIES OF INPUT-OUTPUT NETWORKS
}

Ernest Liu

Aleh Tsyvinski

Working Paper 28178

http://www.nber.org/papers/w28178

\author{
NATIONAL BUREAU OF ECONOMIC RESEARCH \\ 1050 Massachusetts Avenue \\ Cambridge, MA 02138 \\ December 2020
}

We thank Daron Acemoglu, Ruben Enikolopov, Benny Kleinman, Stephen Redding, and Stefan Steinerberger for comments, and Yinshan Shang and Alexander Zimin for research assistance. The views expressed herein are those of the authors and do not necessarily reflect the views of the National Bureau of Economic Research.

NBER working papers are circulated for discussion and comment purposes. They have not been peer-reviewed or been subject to the review by the NBER Board of Directors that accompanies official NBER publications.

(C) 2020 by Ernest Liu and Aleh Tsyvinski. All rights reserved. Short sections of text, not to exceed two paragraphs, may be quoted without explicit permission provided that full credit, including $(\odot$ notice, is given to the source. 
Dynamical Structure and Spectral Properties of Input-Output Networks

Ernest Liu and Aleh Tsyvinski

NBER Working Paper No. 28178

December 2020

JEL No. E0,E23

\begin{abstract}
$\underline{\text { ABSTRACT }}$
We associate a dynamical system with input-output networks and study its spectral properties. Specifically, we develop a dynamic production network model featuring adjustment costs of changing inputs and thus gradual recovery from temporary TFP shocks. First, we explicitly solve for the output and welfare effects of temporary shocks. We show shocks to sectors that generate significant sales through distant linkages to the consumer are most damaging. Second, we eigendecompose the input-output matrix and show, because higher-order linkages take longer to recover, fewer eigenvectors are needed to represent the welfare impact of sectoral shocks in the dynamic economy compared to the Domar weights. Third, we analyze the U.S. input-output structure and show the welfare impact of temporary shocks has a low-dimensional, 4-factor structure (out of 171 eigenvectors). Finally, we revisit the historical use of input-output analysis in target selection for bombing Nazi Germany and Imperial Japan during WWII.
\end{abstract}

Ernest Liu

Princeton University

Department of Economics

Julis Romo Rabinowitz Building

Princeton, NJ 08544

ernestliu@princeton.edu

Aleh Tsyvinski

Department of Economics

Yale University

Box 208268

New Haven, CT 06520-8268

and NBER

a.tsyvinski@yale.edu 


\section{Introduction}

The study of production networks recently became an active research agenda in macroeconomics, reviving and developing the classic analysis of Leontief. The most common setting is that of the static general equilibrium environment subject to sectoral shocks.

The recent theoretical advances in the analysis of networks and graphs take an inherently dynamical perspective. Spectral graph theory (Chung (1997), Grigor'yan (2018), Spielman (2019)) associates a dynamical system, typically a diffusion operator, with the static network and studies the eigenvectors and eigenvalues of the Laplacian. The Leontief-inverse matrix in the input-output analysis is the inverse of the Laplacian of the network and thus admits a parallel analysis. Similarly, a classical way to analyze a non-negative matrix (Berman and Plemmons (1994)), which is the input-output network, is by associating with it a continuous time dynamical system, or a flow, represented by a system of differential equations. ${ }^{1}$ This system has an explicit solution in terms of matrix exponentials and hence the properties of solutions are intimately related to the properties of the matrix (Colonius and Kliemann (2014)). In particular, the eigenvalues and eigenvectors of the input-output matrix determine the evolution of the dynamical system and thus can be used for the analysis of shock propagations. Our main methodological aim is to bring the techniques of dynamical systems and spectral graph theory to the analysis of production networks.

In this paper, such a dynamical system arises from a dynamic model of production networks in which there are costs for changing inputs of production. Following a temporary negative shock, adjustment costs lead to a gradual movement of the economy to the steady state. While the steady state of the economy coincides with the static model, the transition path of the dynamic transmission of shocks across sectors is in general different from the standard setting and the temporary shocks have lasting impact. Our primary goal is to characterize the dynamic path of the propagation of shocks through the input-output linkages and the determinants of the output trajectory and welfare.

Specifically, we extend the model of Acemoglu, Carvalho, Ozdaglar, and Tahbaz-Salehi (2012) and Jones $(2011,2013)$ by introducing a cost of upward change of inputs that depends on the speed of adjustment. The standard model corresponds to the case of zero adjustment costs and thus an immediate adjustment to the temporary shocks. For positive adjustment costs, the standard model is a steady state of our dynamic model. We show that analysis of the determinants of the flow of output (that is, the solution to the dynamical system of differential equations) is closely related to the properties of the static input-output matrix

\footnotetext{
${ }^{1}$ The behavior of the Markov chain on the input-output network is a closely related dynamical system (Kemeny and Snell (1960)).
} 
as in the mathematical approaches described above. The analysis of welfare impact of temporary shocks also adds new considerations in analyzing the temporal structure of the flow.

We first characterize the transition path of the sectoral outputs and consumptions. Even when the sectoral TFP recovery is immediate, due to adjustment costs, the use of intermediate goods cannot jump instantaneously and only recovers gradually. The gradual recovery of the use of inputs then translates into the gradual recovery of the output in sectors that use those inputs. As the transition takes time, the speed of which is determined by the magnitude of the adjustment costs, the path of adjustment has non-trivial welfare implications for a consumer who discounts the future. We thus next characterize the impact of the sectoral TFP shock on consumer welfare. We show that the elasticity of welfare to temporary negative TFP shocks is proportional to the difference between two terms. The first is the sectoral Domar weight that captures the welfare impact of a permanent negative TFP shock. The second term captures the effects of the slow recovery of the production network. This term has a similar form to the Domar weight but where the power series of the subsequent round of effects on production is discounted by the product of the consumer's discount rate and the adjustment cost parameter. Temporary shocks have lasting impact on the output and welfare precisely because input-output linkages take time to recover. These higher-order linkages are represented by the series of the powers of input-output matrix. Importantly, the difference between these two terms disproportionately removes the lower-order rounds of the production effects while keeping the tail entries unchanged.

For example, one can contrast two different networks. In a horizontal network where there are no input-output linkages, the adjustment costs are irrelevant, and a negative TFP shock has zero impact on the economy once the TFP reverts. In a vertical economy, where sectors are ordered and each supplies only to the next one, in contrast, temporary shocks have a lasting impact, and the damage is more severe when the shocked sector is more distant from the final consumer.

We then show that the welfare measure is similar to the concept of alpha centrality in the network literature with the difference that the weights increase for the higher-order linkages. One can view this also as a multi-scale representation of the economy. We show that the importance of the higher-order links is determined by a parameter that is a combination of the speed of adjustment and the discount rate of the consumer. This one-parameter family of economies then shows the importance of temporary shocks' local versus global effects. That is, the economy is represented at different scales or the levels of coarseness spotlighting the relative importance of the higher-order links and thus the importance of the global versus local structures. 
Summarizing our first main result of the paper: in the presence of adjustment frictions, we derive the explicit representation for the output and welfare effects of the temporary shocks. Specifically, the shocks to sectors that generate significant sales through distant linkages to the consumer are disproportionately damaging to the economy.

Our second set of results is the characterization of the main driving forces of the welfare impact of temporary shocks. Since we have shown the importance of the higher-order production links, this naturally leads us to analyze the spectral or eigendecomposition of the input-output matrix - its eigenvectors and eigenvalues. The main reason for this is that once the matrix is diagonalized, the powers of it, which represent the higher order production links, take a particularly simple form.

The concise decomposition of the welfare effects of shocks as a combination of the eigenvectors and the power series of the eigenvalues is our second main theoretical result. Specifically, consider a temporary shock vector that is itself an eigenvector. The impact of the shock along the entire transition path becomes a continuously decayed version of the initial shock, with rate of decay governed by the eigenvalue. An important corollary of this logic shows a marked contrast with the eigendecomposition of the Domar weights, and thus with the static economy. Because dynamic adjustment costs significantly down-weight the direct and initial rounds of network effects, our model effectively up-weights the higher powers in the power series of eigenvalues, thereby up-weighting the relative importance for the shock profiles with greater eigenvalues and down-weighting the shock profiles with the lower eigenvalues. This implies that fewer eigenvalues are needed to represent the welfare impact of the shocks in a dynamic economy. In other words, the dynamic economy may have a factor structure where the small set of factors can capture the importance of temporary shocks. In contrast, the Domar weights may be significantly higher dimensional: because the Domar weights do not discount the direct and initial rounds of network effects, even eigenvectors with small eigenvalues may have a sizable contribution in explaining TFP shocks in the static model. In summary, sectoral shocks may not have a low-dimensional representation in the static model but may have one in our dynamic model. The concise representation of complex high dimensional systems via a few reduced coordinates is also the primary goal of the well developed literature on nonlinear dimensionality reduction using spectral methods (e.g., Coifman, Kevrekidis, Lafon, Maggioni, and Nadler (2008)).

Our third set of results is an empirical analysis of the eigendecomposition of the U.S. input-output structure. We first show that 95 percent of the welfare effect of temporary sectoral shocks can be represented by only four eigenvectors. That is, the U.S. input-output network has a very low- dimensional (4-factor) structure. In contrast, for the Domar weight almost all of the 171 eigenvectors are important, and, hence, the Domar weight is a high- 
dimensional object. Because input-output tables are not symmetric, the eigenvectors are not orthogonal to each other. In fact, many eigenvectors are correlated, thereby picking shocks to the same groups of sectors. We identify the groups of sectors that form the four factors. The first eigenvector represents shocks to the heavy manufacturing sectors. The second eigenvector strongly and negatively correlates with the first and represents three groups of industries: (1) most notably, the two sectors relating to agencies, brokerages, and insurance; (2) manufacturing of consumer goods; (3) it has negative entries on the heavy manufacturing industries, partly neutralizing the shock profile from the first eigenvector. The third eigenvector correlates positively with the second eigenvector and has positive entries on the manufacturing of consumer goods and chemicals. The fourth eigenvector has closeto-zero correlations with the previous three eigenvectors. The main sector picked is radio and television broadcasting; in addition, it also has negative entries on the manufacturing of chemicals, plastic, and rubber products, partly neutralizing the shock profiles represented by the third eigenvector. Summarizing, we find that the welfare impact of any negative temporary shocks can be represented by only four (out of 171) eigenvectors.

Our fourth set of results is based on revisiting one of the earliest historical applications of the input-output analysis. During the World War II, Wassily Leontief was part of the small groups of economist that used input-output analysis for target selection for strategic bombing (Guglielmo (2008), Harrison (2020)). We use the input-output table of pre-war Nazi Germany and Imperial Japan to parallel that analysis. First, we provide the list of sectors to which temporary shocks generate the largest impact. Second, we show, for the purpose of finding vulnerability to temporary shocks, both of these input-output tables also exhibit low-dimensional representations: the first three eigenvectors explain $92 \%$ of the variation in welfare losses for Japan and 85\% for Germany. Third, we demonstrate the over-time impact of shocks to each sector on every other sector of the economy, and we show shocks to the metal sectors tend to have lasting damage across both pre-WWII Germany and Japan.

We now briefly summarize the literature. There is a modern revival of the literature on production networks (see, e.g., reviews in macroeconomics of Carvalho (2014), Carvalho and Tahbaz-Salehi (2019), and Grassi and Sauvagnat (2019); and, more broadly, Bloch, Jackson, and Tebaldi (2020) and Jackson, Rogers, and Zenou (forthcoming)). Acemoglu, Carvalho, Ozdaglar, and Tahbaz-Salehi (2012) show idiosyncratic sectoral productivity shocks may have aggregate impact. Jones $(2011,2013)$ develops a model of production networks with distortions. A number of recent papers develop various important aspects of the macroeconomic implications of the input-output and production structure of the economy: for example, Acemoglu, Akcigit, and Kerr (2015), Oberfield (2018), Baqaee (2018), Liu (2019), Baqaee and Farhi (2019, 2020), Bigio and La'O (2020), and Golub, Elliot, and Leduc (2020). 
Unlike these papers, which all feature static models, our main contribution is to study the dynamic adjustment of the economy with the adjustment costs through the lens of the spectral graph theory and dynamical system theory. ${ }^{2}$ We find that temporary shocks to sectors that generate significant sales through distant linkages to the consumer are disproportionately damaging to the economy. We also find that shocks to upstream sectors are especially damaging relative to the size of these sectors, precisely because higher order linkages take a long time to recover. Our theory also derives a precise notion of upstreamness that relates to but differs from the upstreamness measure of Antràs, Chor, Fally, and Hillberry (2012) and the distortion centrality measure of Liu (2019). Our analysis of disrupting the Axis economies is inspired by an important paper of Davis and Weinstein (2002). The closest in this aspect to our work is the study of the effects of the 2011 Japanese earthquake on the supply chains by Carvalho, Nirei, Saito, and Tahbaz-Salehi (forthcoming) set in the static framework and an exceptionally detailed study of the effects of bombing Germany on resistance to Nazis (Adena, Enikolopov, Petrova, and Voth (2020)).

\section{Model}

Our model is a dynamic Cobb-Douglas production network. We extend the standard, static production network model of Acemoglu et al. (2012) and Jones (2011, 2013) by introducing dynamic adjustment costs in input-output linkages. As we show below, allocations in the equilibrium of the static model coincide with our dynamic economy's steady-state but differ from our transitional path.

There is a representative consumer with exogenous labor supply $\bar{\ell}$ and $N$ production sectors that produce from labor and intermediate inputs. The consumer has utility

$$
V \equiv \int_{0}^{\infty} e^{-\rho t} \ln c(t) d t
$$

where $c(t)$ is a Cobb-Douglas aggregator over sectoral goods $j=1, \ldots, N$ :

$$
c(t)=\chi_{c} \prod_{j=1}^{N}\left(c_{j}(t)\right)^{\beta_{j}}, \quad \sum_{j=1}^{N} \beta_{j}=1
$$

where $\chi_{c} \equiv \prod_{j=1}^{N} \beta_{j}^{-\beta_{j}}$ is a normalizing constant. We refer to $c(t)$ as aggregate consumption and GDP interchangeably.

\footnotetext{
${ }^{2}$ In a different setting, Steinerberger and Tsyvinski (2019) associate a dynamical system with the static model of optimal taxation.
} 
At each time $t$, the output of production sector $i$ satisfies

$$
q_{i}(t)=\chi_{i} z_{i}(t)\left(\ell_{i}(t)\right)^{\alpha_{i}} \prod_{j=1}^{N}\left(m_{i j}(t)\right)^{\sigma_{i j}}, \quad \sum_{j=1}^{N} \sigma_{i j}+\alpha_{i}=1
$$

where $0 \leq \alpha_{i}, \sigma_{i j} \leq 1, \chi_{i} \equiv \alpha_{i}^{-\alpha_{i}} \prod_{j=1}^{N} \sigma_{i j}^{-\sigma_{i j}}$ is a normalizing constant, $z_{i}(t)$ is sectoral total factor productivity, $l_{i}(t)$ is the amount of labor used, and $m_{i j}(t)$ is the amount of the intermediate good of the sector $j$ used in the production of the good $i$.

From now on, wherever it does not cause confusion, we suppress dependence on time $t$ in the notation.

Our departure from the standard model lies in how intermediate inputs $m_{i j}$ are delivered from seller $j$ to buyer $i$. In the standard model, as the economy's fundamentals change at time $t$, the prices and quantities in all connected sectors adjust immediately and fully to their new equilibrium levels all at the same time $t$.

We introduce the concept of time through the transportation of intermediate inputs across producers. Our formulation captures the notion that, following temporary negative shocks, the recovery of input-output linkages must be gradual, and temporary shocks therefore may have lasting impact on the economy. Specifically, to use input quantity $m_{i j}$ at time $t$, sector $i$ needs to buy

$$
m_{i j} \times \exp \left(\delta \dot{m}_{i j} / m_{i j} \times \mathbf{1}\left(\dot{m}_{i j}>0\right)\right)
$$

units of input $j$. The term $\dot{m}_{i j} \equiv d m_{i j}(t) / d t$ is the rate of change in the quantity of intermediate input $j$ used by sector $i$ and the term $\mathbf{1}\left(\dot{m}_{i j}>0\right)$ states that only the increases in the goods use matter. The term $\exp \left(\delta \dot{m}_{i j} / m_{i j} \times \mathbf{1}\left(\dot{m}_{i j}>0\right)\right)$ captures sluggish upward adjustment of inputs and can be interpreted as an iceberg cost that producer $i$ has to incur when it raises the quantity of input $j$. The parameter $\delta$ captures the ease of adjustment; when $\delta \rightarrow 0$, adjustment costs vanish.

The market clearing conditions are

$$
\begin{gathered}
q_{j}=c_{j}+\sum_{i} m_{i j} \exp \left(\delta \dot{m}_{i j} / m_{i j} \times \mathbf{1}\left(\dot{m}_{i j}>0\right)\right) \text { for all } j, \\
\bar{\ell}=\sum_{i} \ell_{i} .
\end{gathered}
$$

For simplicity, we assume goods delivered to the consumer are not subject to adjustment costs, and neither is the use of labor across production sectors. These choices are made for expositional simplicity and are without loss of generality. We can always accommodate adjustment costs in the purchase of labor or the consumption good by creating a fictitious 
production sector that buys the consumption bundle and sells to the consumer or buys labor and sells to other producers.

Equilibrium and Steady State All producers are price-takers. Let

$$
\Xi(t) \equiv\left\{m_{i j}(t), c_{j}(t), q_{j}(t), \ell_{j}(t), c(t)\right\}_{i, j=1}^{N}
$$

be the input-output quantity allocation at time $t$, let $\mathcal{P}(t) \equiv\left\{p_{j}(t), w(t)\right\}_{j=1}^{N}$ be the set of sectoral prices and of wages at time $t$, and let $\boldsymbol{z}(t) \equiv\left\{z_{j}(t)\right\}_{j=1}^{N}$ denote the set of time- $t$ productivities. Given the initial condition $\Xi(0)$ and the sequence of sectoral productivity $Z(\cdot)$, an equilibrium is the sequence of allocation and prices $\Xi(t), \mathcal{P}(t)$ such that all producers choose input bundles to minimize costs, with

$$
\begin{gathered}
p_{i}=\frac{1}{z_{i}} w^{\alpha_{i}}\left(p_{j} \exp \left(\delta \dot{m}_{i j} / m_{i j} \times \mathbf{1}\left(\dot{m}_{i j}>0\right)\right)\right)^{\sigma_{i j}}, \\
p_{j} m_{i j} \exp \left(\delta \dot{m}_{i j} / m_{i j} \times \mathbf{1}\left(\dot{m}_{i j}>0\right)\right)=\sigma_{i j} p_{i} q_{i}, \\
w \ell_{i}=\alpha_{i} p_{i} q_{i}, \quad p_{j} c_{j}=\beta_{j} c .
\end{gathered}
$$

We normalize the consumer price index to one: $1=\prod_{j=1}^{N} p_{j}^{\beta_{j}}$. A steady-state equilibrium is one in which $\boldsymbol{z}(t), \Xi(t), \mathcal{P}(t)$ are all time invariant.

In what follows, we use boldface to denote vectors (lower case) and matrices (upper case). Let $\boldsymbol{\Sigma} \equiv\left[\sigma_{i j}\right]$ denote the matrix of input-output expenditure shares, and let $\boldsymbol{\beta}$ denote the $N \times 1$ vector of consumption expenditure shares. Let $\boldsymbol{\alpha}$ be the vector of sectoral value-added shares. Let $\boldsymbol{\gamma}^{\prime} \equiv \boldsymbol{\beta}^{\prime}(\boldsymbol{I}-\boldsymbol{\Sigma})^{-1}$ be the vector of Domar weights, i.e., sectoral sales relative to GDP.

Discussion When $\delta=0$, the economy does not feature adjustment costs, and the model becomes a repeated version of the static economy in Acemoglu et al. (2012): given the vector of sectoral TFP $\boldsymbol{z}(t)$ at each time $t$, the $\log$-GDP is $\ln c(t)=$ const $+\boldsymbol{\gamma}^{\prime} \ln \boldsymbol{z}(t)$, where $\boldsymbol{\gamma}$ is the vector of Domar weights.

When $\delta>0$, the economy features adjustment costs. However, allocations and prices in the steady state of this dynamic model coincide with those the static equilibrium in Acemoglu et al. (2012). The Hulten's theorem holds across steady-states: the sales share $\gamma_{i}$ of sector $i$ characterizes the steady-state importance of each sector's TFP. Specifically, let $c^{s s}$ denote the steady-state consumption per period; then

$$
\ln c^{s s}=\text { const }+\gamma^{\prime} \ln \boldsymbol{z}
$$


In our formulation, input usage is slow to expand but may shrink instantaneously. This is for expositional simplicity; the model can be easily generalized to accommodated sluggish downward adjustment of inputs as well.

\section{Slow recovery from a temporary TFP shock}

Consider a production network affected by temporary negative TFP shocks to some sectors. These shocks reduce sectoral production and may propagate through input-output linkages and affect output in other sectors. After these negative shocks revert, how quickly does the economy recover? We show, when production linkages take time to recover, the topology of a production network is a key determinant of its resilience to negative shocks.

Figure 1: Two stylized example networks

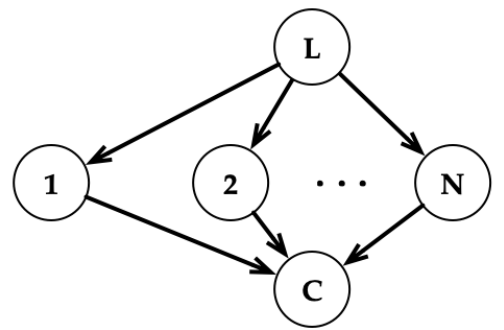

(a) A horizontal production economy

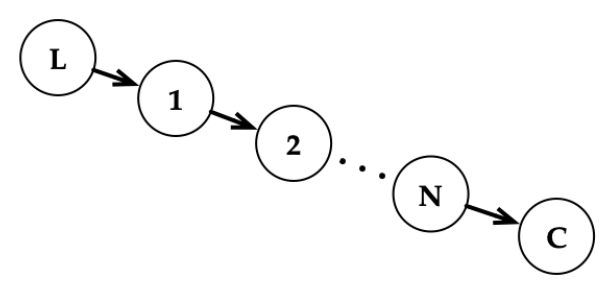

(b) A vertical production chain

To illustrate the intuition, consider two example networks. In Figure 1, panel (a) shows the network structure of a horizontal production economy. Here, labor is the only factor of production in each sector $i \in\{1, \ldots, N\}$, and sectors $i$ does not use any of the goods $j \in\{1, \ldots, N\}$ in production. All of the goods are part of the consumption bundle $c$. Since there are no input-output linkages, the adjustment costs in this setting are irrelevant, and a negative TFP shock to $z_{i}$ has zero impact on the economy once the TFP reverts back, measured by either sectoral output or GDP.

Now consider panel (b), which shows the network structure of a vertical production chain. Here, labor is the only factor of production of sector 1 , and each subsequent sector $i$ uses inputs only of the sector $i-1$. Only good $N$ is used in final consumption $c$. Consider a temporary decline in sector 1's productivity $z_{1}$. Sector 1's output declines for the duration of the negative shock; moreover, because sector 2 requires good 1 as inputs, the output of sector 2 declines as well, and in fact output declines in all sectors $i \in\{1, \ldots, N\}$. After the initial TFP shock disappears and as $z_{1}$ reverts, output in sector 1 recovers immediately. However, because of adjustment costs in the recovery of input-output linkages, sectoral output for all $i \geq 2$ may stay extendedly depressed, and the economy as a whole-measured by the 
consumption aggregator $c(t)$, i.e., the GDP - may take a long time to recover. By contrast, a temporary reduction in sector $N$ 's $\operatorname{TFP} z_{N}$ has no lasting impact on the economy, which recovers immediately after the shock dissipates. More generally, in the vertical network of panel (b), the economy recovers more slowly from negative shocks that affect relatively upstream sectors.

We now formalize the analysis, and we analyze sectoral susceptibility in a production network from our dynamic perspective.

\subsection{Negative shocks and transitional dynamics}

We analyze an economy initially in a steady-state with sectoral $\log$-productivities $\left\{\ln z_{i}\right\}_{i=1}^{N}$, and we consider temporary, negative TFP shocks that reduce sectoral productivities at time zero to $\left\{\ln z_{i}-\tilde{z}_{i}\right\}_{i=1}^{N}$. We use $\tilde{z}_{i}>0$ to denote the absolute value in $\operatorname{logs}$ of the negative shocks for sector $i$. For expositional simplicity, we assume sectoral TFP reverts back instantaneously to the pre-shock steady-state levels $\left\{z_{i}\right\}_{i=1}^{N}$. We use $t=0^{-}$and $t=0$ to respectively index the time at and after the negative TFP shocks. When the negative shocks are present at $t=0^{-}, \log -G D P$ declines by $\gamma^{\prime} \tilde{\boldsymbol{z}}$ relative to its steady-state level, where $\gamma^{\prime}$ is the Domar weights, consistent with Hulten (1978) and Acemoglu et al. (2012). We now analyze the dynamic path of sectoral output and GDP during the recovery, from $t=0$ onwards.

Even as sectoral TFP recovers at $t=0$, the use of intermediate inputs can only grow gradually over time and cannot jump instantaneously. Hence, sectoral output increases exactly in proportion to the TFP recovery, and the total output in sector $j$ exceeds the total quantity of good $j$ used as production inputs. The excess output is dispensed as the adjustment costs required to expand input $j$ for the future. With passage of time, sectors continue to expand the use of inputs, sectoral output continues to expand even though TFP is constant. Eventually the economy converges back to the initial steady-state as $t \rightarrow \infty$.

To solve for the transition path, let

$$
x_{j}(t) \equiv \ln \frac{q_{j}(t)-c_{j}(t)}{\sum_{i} m_{i j}(t)}
$$

be the log-ratio between the total quantity of good $j$ supplied to and used by other producers. The ratio $\frac{q_{j}(t)-c_{j}(t)}{\sum_{i} m_{i j}(t)}$ is equal to one in a steady-state, and $x_{j}=0$ for all $j$. Away from a steadystate, the ratio captures the proportional adjustment costs incurred for expanding input $j$ in production. Because all producers $i$ spend a constant share of input expenditure on good $j$ along the transition path, $x_{j}(t)=\delta \dot{m}_{i j} / m_{i j}$ for all $i$, and $\delta^{-1} x_{j}(t)$ captures the rate at 
which all sectors expand their use of input $j$.

Proposition 1. Laws of Motion. Consider a TFP shock vector $\tilde{\boldsymbol{z}}$ that affects the steadystate economy at time zero and reverts back instantaneously. The law of motion for sectoral output vector $\boldsymbol{q}$ is

$$
\frac{\mathrm{d} \ln \boldsymbol{q}}{\mathrm{d} t}=\delta^{-1} \boldsymbol{\Sigma} \boldsymbol{x}(t), \quad \text { with the initial condition } \ln \boldsymbol{q}(0)=\ln \boldsymbol{q}^{s s}-\boldsymbol{\Sigma}(\boldsymbol{I}-\boldsymbol{\Sigma})^{-1} \tilde{\boldsymbol{z}}
$$

where $\boldsymbol{I}$ is the identity matrix and $\boldsymbol{\Sigma} \equiv\left[\sigma_{i j}\right]$ is the matrix of input-output coefficients.

The law of motion for GDP is

$\frac{\mathrm{d} \ln c}{\mathrm{~d} t}=\delta^{-1} \boldsymbol{\beta}^{\prime} \boldsymbol{\Sigma} \boldsymbol{x}(t), \quad$ with the initial condition $\ln c(0)=\ln c^{s s}-\boldsymbol{\beta}^{\prime} \boldsymbol{\Sigma}(\boldsymbol{I}-\boldsymbol{\Sigma})^{-1} \tilde{\boldsymbol{z}}$.

The dynamic path of $\boldsymbol{x}$ satisfies the ODE system

$$
\frac{d \boldsymbol{x}(t)}{d t}=-(\boldsymbol{I}-\boldsymbol{\Sigma}) \boldsymbol{x}(t), \quad \text { with the initial condition } \boldsymbol{x}(0)=\tilde{\boldsymbol{z}}
$$

To understand this Proposition, first suppose the negative TFP shocks were permanent. Output declines in sectors directly affected by the shocks. Moreover, because of production linkages, output also declines in sectors that purchase inputs - directly or indirectly - from the shocked sectors. The total impact of negative shocks on sectoral output is captured by $-(\boldsymbol{I}-\boldsymbol{\Sigma})^{-1} \tilde{\boldsymbol{z}}$, where the Leontief inverse $(\boldsymbol{I}-\boldsymbol{\Sigma})^{-1} \equiv \boldsymbol{I}+\boldsymbol{\Sigma}+\boldsymbol{\Sigma}^{2}+\cdots$ captures the infinite rounds of higher order effects through input-output linkages. This is indeed the finding of Acemoglu et al. (2012) and Acemoglu et al. (2015) in the standard, static production network model.

This Proposition 1 instead pertains to temporary shocks. As sectoral TFP recovers instantaneously, log-output directly recovers by $\tilde{\boldsymbol{z}}$; hence, at time $t=0$, sectoral output satisfies

$$
\ln \boldsymbol{q}(0)=\underbrace{\ln \boldsymbol{q}^{s s}}_{\text {initial steady state }}-\underbrace{(\boldsymbol{I}-\boldsymbol{\Sigma})^{-1} \tilde{\boldsymbol{z}}}_{\begin{array}{c}
\text { effect of permanent } \\
\text { negative shocks }
\end{array}}+\underbrace{\tilde{\boldsymbol{z}}}_{\text {recovery of TFP }} .
$$

The input-output linkages destroyed by the negative shocks take time to recover. Because $\delta^{-1} x_{j}(t)=\dot{m}_{i j} / m_{i j}$ captures the rate at which all producers expand their use of input $j$, the output of sector $i$ grows at rate

$$
\dot{q}_{i} / q_{i}=\delta^{-1} \sum_{j=1}^{N} \sigma_{i j} x_{j}(t)
$$


and in the vector form

$$
\frac{\mathrm{d} \ln \boldsymbol{q}}{\mathrm{d} t}=\delta^{-1} \boldsymbol{\Sigma} \boldsymbol{x}(t) .
$$

The law of motion for GDP follows from the fact that $\ln c(t)-\ln c^{s s}=\boldsymbol{\beta}^{\prime}\left(\ln \boldsymbol{q}(t)-\ln \boldsymbol{q}^{s s}\right)$ for all $t$.

Finally, to derive the law of motion for $\boldsymbol{x}(t)$, note

$$
\frac{\mathrm{d} x_{j}(t)}{\mathrm{d} t}=\frac{\mathrm{d} \ln q_{j}}{\mathrm{~d} t}-\frac{\mathrm{d} \ln \left(\sum_{i} m_{i j}(t)\right)}{\mathrm{d} t}
$$

captures the difference between the rate at which sectoral output expands (the first term) and the rate at which sectoral goods are used as intermediate inputs (the second term); the latter is equal to $\delta^{-1} x_{j}(t)$. In the vector form,

$$
\frac{\mathrm{d} \boldsymbol{x}}{\mathrm{d} t}=\delta^{-1} \boldsymbol{\Sigma} \boldsymbol{x}-\delta^{-1} \boldsymbol{x}
$$

The ODE system for $\boldsymbol{x}(t)$ has an explicit solution in terms of the matrix exponential:

$$
\boldsymbol{x}(t)=e^{-\delta^{-1}(\boldsymbol{I}-\boldsymbol{\Sigma}) t} \tilde{\boldsymbol{z}},
$$

where matrix exponential for any generic matrix $\boldsymbol{M}$ is defined as

$$
e^{M} \equiv \sum_{k=0}^{\infty} \frac{\boldsymbol{M}^{k}}{k !}
$$

Intuitively, immediately after the negative TFP shock recedes, $\boldsymbol{x}(0)=\tilde{\boldsymbol{z}}$. As production linkages recover over time and as the economy converges back to the steady-state, $\boldsymbol{x}(t)$ converges to the zero vector. The term $\delta$ modulates the rate of convergence; the system converges at a faster rate if adjustment $\operatorname{cost} \delta$ is small. The next proposition describes the time paths or the flow of the sectoral outputs and consumption.

Proposition 2. Flow of Output and Consumption. The flow of sectoral output satisfies

$$
\ln \boldsymbol{q}(t)=\ln \boldsymbol{q}^{s s}-\boldsymbol{\Sigma}(\boldsymbol{I}-\boldsymbol{\Sigma})^{-1} e^{-\delta^{-1}(\boldsymbol{I}-\boldsymbol{\Sigma}) t} \tilde{\boldsymbol{z}}
$$

and the flow of aggregate consumption satisfies

$$
\ln c(t)=\ln c^{s s}-\boldsymbol{\beta}^{\prime} \boldsymbol{\Sigma}(\boldsymbol{I}-\boldsymbol{\Sigma})^{-1} e^{-\delta^{-1}(\boldsymbol{I}-\boldsymbol{\Sigma}) t} \tilde{\boldsymbol{z}} .
$$

Proof. We have 


$$
\begin{aligned}
\ln \boldsymbol{q}(t) & =\boldsymbol{q}(0)+\delta^{-1} \boldsymbol{\Sigma} \int_{0}^{t} \boldsymbol{x}(s) d s \\
& =\boldsymbol{q}(0)+\delta^{-1} \boldsymbol{\Sigma}\left[\int_{0}^{t} e^{-\delta^{-1}(\boldsymbol{I}-\boldsymbol{\Sigma}) s} d s\right] \tilde{\boldsymbol{z}} \\
& =\underbrace{\ln \boldsymbol{q}^{s s}-(\boldsymbol{I}-\boldsymbol{\Sigma})^{-1} \tilde{\boldsymbol{z}}+\tilde{\boldsymbol{z}}}_{\boldsymbol{q}(0)}+\boldsymbol{\Sigma}(\boldsymbol{I}-\boldsymbol{\Sigma})^{-1}\left(\boldsymbol{I}-e^{-\delta^{-1}(\boldsymbol{I}-\boldsymbol{\Sigma}) t}\right) \tilde{\boldsymbol{z}} \\
& =\ln \boldsymbol{q}^{s s}-\boldsymbol{\Sigma}(\boldsymbol{I}-\boldsymbol{\Sigma})^{-1} e^{-\delta^{-1}(\boldsymbol{I}-\boldsymbol{\Sigma}) t} \tilde{\boldsymbol{z}} .
\end{aligned}
$$

The expression for $c(t)$ is derived analogously.

When productivity in sector $j$ recovers, the sector's output expands immediately, which gradually translates into the expansion of input $j$ used in other sectors $i$, thereby causing $i$ 's output to expand gradually over time. The vector $\left(-(\boldsymbol{I}-\boldsymbol{\Sigma})^{-1} \tilde{\boldsymbol{z}}+\tilde{\boldsymbol{z}}\right)=-\boldsymbol{\Sigma}(\boldsymbol{I}-\boldsymbol{\Sigma})^{-1} \tilde{\boldsymbol{z}}$ captures the extent to which log-sectoral outputs at $t=0$ are below their steady-state levels; it can be re-written as

$$
-\boldsymbol{\Sigma}(\boldsymbol{I}-\boldsymbol{\Sigma})^{-1} \tilde{\boldsymbol{z}}=-\left(\sum_{s=1}^{\infty} \boldsymbol{\Sigma}^{s}\right) \tilde{\boldsymbol{z}}
$$

where each successive term in the summation captures a higher round of input-output linkages to be recovered from the initial shock. The expression $\left(-\boldsymbol{\Sigma}(\boldsymbol{I}-\boldsymbol{\Sigma})^{-1} e^{-\delta^{-1}(\boldsymbol{I}-\boldsymbol{\Sigma}) t}\right)$ is the log-deviation in output relative to steady-state levels at time $t$; it is the continuous time analogue of the discrete partial sum $-\sum_{s=t}^{\infty} \Sigma^{s}$. By varying $t$, the expression captures the fact that general equilibrium linkages recover gradually, and higher rounds of linkages take longer to recover.

The rate of recovery is inversely related to $\delta$. As $\delta \rightarrow 0$, the convergence towards the steady-state becomes instantaneous:

$$
\lim _{\delta \rightarrow 0}\left(-\boldsymbol{\Sigma}(\boldsymbol{I}-\boldsymbol{\Sigma})^{-1} e^{-\delta^{-1}(\boldsymbol{I}-\boldsymbol{\Sigma}) t}\right)=\mathbf{0} \quad \text { for any } t>0
$$

More broadly, this proposition shows that the properties of the dynamical system described by the gradual adjustment of the economy are tightly related to the properties of the input-output matrix via the sequence of its powers $\Sigma^{s}$. The parameter $\delta$ modulates the speed of adjustment and thus makes the dynamical system that we study somewhat broader than the classical dynamical systems that are associated with a given matrix $\boldsymbol{A}$. There, a typical dynamical system is given by $\boldsymbol{b}=\boldsymbol{A} \boldsymbol{b}$ (Colonius and Kliemann (2014)). 


\subsection{Sectoral shocks and welfare}

We now characterize the impact of sectoral TFP shocks on consumer welfare. Let $V^{s s}$ denote consumer welfare in the initial steady state absent the TFP shock.

Proposition 3. Welfare Impact of Temporary TFP Shocks. Let

$$
\boldsymbol{v}^{\prime} \equiv \frac{1}{\rho}\left[\boldsymbol{\beta}^{\prime}(\boldsymbol{I}-\boldsymbol{\Sigma})^{-1}-\boldsymbol{\beta}^{\prime}\left(\boldsymbol{I}-\frac{\boldsymbol{\Sigma}}{1+\rho \delta}\right)^{-1}\right] .
$$

The impact of temporary, negative TFP shocks $\tilde{\boldsymbol{z}}$ on welfare is

$$
V(\tilde{\boldsymbol{z}})-V^{s s}=\int_{0}^{\infty} e^{-\rho s}\left(\ln c(s)-\ln c^{s s}\right) d s=-\boldsymbol{v}^{\prime} \tilde{\boldsymbol{z}}
$$

Proof. We have

$$
\begin{aligned}
V(\tilde{\boldsymbol{z}})-V_{0}^{s s} & =\int_{0}^{\infty} e^{-\rho s}\left(\ln c(s)-\ln c_{0}^{s s}\right) d s \\
& =-\boldsymbol{\beta}^{\prime} \boldsymbol{\Sigma}(\boldsymbol{I}-\boldsymbol{\Sigma})^{-1} \int_{0}^{\infty} e^{-\delta^{-1}((1+\rho \delta) \boldsymbol{I}-\boldsymbol{\Sigma}) t} d t \tilde{\boldsymbol{z}} \\
& =-\delta \boldsymbol{\beta}^{\prime} \boldsymbol{\Sigma}(\boldsymbol{I}-\boldsymbol{\Sigma})^{-1}((1+\rho \delta) \boldsymbol{I}-\boldsymbol{\Sigma})^{-1} \tilde{\boldsymbol{z}} \\
& =-\frac{1}{\rho} \boldsymbol{\beta}^{\prime} \boldsymbol{\Sigma}\left[(\boldsymbol{I}-\boldsymbol{\Sigma})^{-1}-((1+\rho \delta) \boldsymbol{I}-\boldsymbol{\Sigma})^{-1}\right] \tilde{\boldsymbol{z}} \\
& =-\frac{1}{\rho}\left[\boldsymbol{\beta}^{\prime}(\boldsymbol{I}-\boldsymbol{\Sigma})^{-1}-\boldsymbol{\beta}^{\prime}\left(\boldsymbol{I}-\frac{\boldsymbol{\Sigma}}{1+\rho \delta}\right)^{-1}\right] \tilde{\boldsymbol{z}} .
\end{aligned}
$$

The vector $\boldsymbol{v}^{\prime}$ captures the elasticity of welfare to temporary, negative TFP shocks. When $\delta=0$, recovery is instantaneous, and temporary shocks have no impact on consumer welfare. The first term, $\frac{1}{\rho} \boldsymbol{\beta}^{\prime}(\boldsymbol{I}-\boldsymbol{\Sigma})^{-1}$, is proportional to the sectoral Domar weight and captures the impact on welfare of permanent negative TFP shocks. The second term $\frac{1}{\rho} \boldsymbol{\beta}^{\prime}\left(\boldsymbol{I}-\frac{\boldsymbol{\Sigma}}{1+\rho \delta}\right)^{-1}$ captures the impact of slow recovery of input-output linkages. One can also think about this term as solving for $\chi^{\prime}$ in the following expression:

$$
\boldsymbol{\beta}^{\prime}+\frac{1}{1+\rho \delta} \Sigma \chi^{\prime}=\chi^{\prime}
$$

which is identical to the expression for the sectoral Domar weights $\left(\boldsymbol{\gamma}^{\prime}\right.$ solves $\left.\boldsymbol{\beta}^{\prime}+\boldsymbol{\Sigma} \boldsymbol{\gamma}^{\prime}=\boldsymbol{\gamma}^{\prime}\right)$ adjusted by the factor $\frac{1}{1+\rho \delta}$. 
It is informative to rewrite $\boldsymbol{v}^{\prime}$ as

$$
\boldsymbol{v}^{\prime}=\frac{1}{\rho} \boldsymbol{\beta}^{\prime} \sum_{s=0}^{\infty}\left(1-(1+\rho \delta)^{-s}\right) \boldsymbol{\Sigma}^{s}
$$

and compare the expression with sectoral Domar weights:

$$
\gamma^{\prime}=\boldsymbol{\beta}^{\prime} \sum_{s=0}^{\infty} \Sigma^{s}
$$

In a static model, the Domar weight captures the impact of sectoral TFP on aggregate consumption, and each term $\boldsymbol{\beta}^{\prime} \Sigma^{s}$ in the power series captures the $s$-th round of network effect: $\boldsymbol{\beta}^{\prime}$ captures the first round, direct effect of TFP on consumption, $\boldsymbol{\beta}^{\prime} \boldsymbol{\Sigma}$ captures the indirect effect of sectoral TFP on other producers who supply to the consumer, and so on. The Domar weight is also equal to a sector's sale relative to GDP, and each term $\boldsymbol{\beta}^{\prime} \boldsymbol{\Sigma}^{s}$ in the power series captures the revenue from the $s$-th round indirect sales to the consumer.

In our dynamic model, temporary shocks may have lasting effect on output and welfare precisely because of higher-order linkages $\Sigma^{s}, s>0$. When $\delta>0$, input-output linkages are slow to recover, and $\left(1-(1+\rho \delta)^{-s}\right) \Sigma^{s}$ captures the present discounted value of consumption affected by the slow recovery of the $s$-th order linkages. Effectively, the power series in (3) disproportionately removes the initial entries in (4) while keeping the tail entries unchanged:

$$
\boldsymbol{v}^{\prime}=\frac{1}{\rho} \boldsymbol{\beta}^{\prime}\left[(1-1) \boldsymbol{\Sigma}^{0}+\left(1-(1+\rho \delta)^{-1}\right) \boldsymbol{\Sigma}^{1}+\left(1-(1+\rho \delta)^{-2}\right) \boldsymbol{\Sigma}^{2}+\ldots\right] .
$$

Note the weight on $\Sigma^{0}$ is 0 and the weight on $\Sigma^{s}$ converges to 1 as $s \rightarrow \infty$.

We now summarize this proposition as our first main result of the paper: in the presence of adjustment frictions, shocks to sectors that generate significant sales through distant linkages to the consumer are disproportionately damaging to the economy. These shocks have large and lasting impact on GDP even as sectoral TFP recovers.

Alpha centrality and global versus local influence We next show that the welfare impact measure $\boldsymbol{v}^{\prime}$ also can be connected to a measure of centrality, alpha centrality, in a network represented by the input-output matrix. The alpha centrality for $\alpha \in(0,1]$ is defined as:

$$
\boldsymbol{\iota}_{\alpha}^{\prime} \equiv \boldsymbol{\beta}^{\prime}(\boldsymbol{I}-\alpha \boldsymbol{\Sigma})^{-1}
$$


Intuitively, this is a centrality measure where a parameter $\alpha$ is used to weigh the higher order input-output linkages, represented by the powers of the matrix $\Sigma$ :

$$
\boldsymbol{\iota}_{\alpha}^{\prime} \equiv \boldsymbol{\beta}^{\prime}\left[\boldsymbol{\Sigma}^{0}+\alpha \boldsymbol{\Sigma}^{1}+\alpha^{2} \boldsymbol{\Sigma}^{2}+\ldots\right]
$$

The $i$-th entry in $\boldsymbol{\beta}^{\prime} \Sigma^{s}$ captures the sales (relative to GDP) that sector $i$ generates through $s$ rounds of linkages before reaching the final consumer.

A related way to think about centrality is in terms of a random walk on the network, where $\boldsymbol{\Sigma}_{i j}$ is the probability of reaching $j$ from $i$ in one walk. The $i j$-th entry in $\Sigma^{s}$ then measures the probability of reaching $j$ from $i$ in the walks of length $s$. As parameter $(\alpha \leq 1)$ decreases, shorter walks become more important, and local influences carry higher significance. When $\alpha$ increases, longer walks become more important, and global influences carry higher significance. In the limit case as $\alpha \rightarrow 1$, the walks of any length carry identical weights, and the alpha centrality measure becomes the Domar weight. In this sense, alpha centrality tunes between rankings based on short walks (local influence) and those based on long walks (global influence) (Benzi and Klymko (2015)).

The welfare $\boldsymbol{v}^{\prime}$ is thus proportional to the difference in the alpha centralities $\boldsymbol{\iota}_{\alpha_{1}}^{\prime}-\boldsymbol{\iota}_{\alpha_{1}}^{\prime}$, where $\alpha_{1}=1$ and $\alpha_{2}=(1+\rho \delta)^{-1}$. Now, let us slightly modify the notion of alpha centrality by defining it as

$$
\tilde{\boldsymbol{\iota}}^{\prime} \equiv \boldsymbol{\beta}^{\prime}\left[a_{0} \boldsymbol{\Sigma}^{0}+a_{1} \boldsymbol{\Sigma}^{1}+a_{2} \boldsymbol{\Sigma}^{2}+\ldots\right]
$$

for some sequence $\left\{a_{0}, a_{1} \ldots\right\}$. Assuming that such weighted power series converge, this measure weights the walks of length $k$ with the parameter $a_{k}$. In the case of alpha centrality with $\alpha<1, a_{k}=\alpha^{k}$ and is geometrically decreasing from $a_{0}=1$ and $a_{\infty}=0$. The welfare measure $\boldsymbol{v}^{\prime}$ is also a (modified version of) alpha centrality with $a_{k}=1-\alpha_{2}^{k}$ and thus increasing between $a_{0}=0$ and $a_{\infty}=1$. One can thus think of it as being conceptually similar to the usual alpha centrality, where the welfare measure, however, relatively prioritizes the longer walks or higher order input output linkages and thus the global over local influences.

The term $(1+\rho \delta)^{-1}$ also defines a one-parameter family of the economies that can be thought of as a multi-scale representation of the static input output matrix. Specifically, the speed of adjustment and the discount factor of the agent determine the scale - the relative importance of the higher-order links and thus the importance of the global versus local structures.

Vertical Economy Revisited. It is now instructive to revisit the examples in Figure 1. In the horizontal economy of panel (a), there are no input-output linkages; consequently, $\boldsymbol{v}$ is the zero vector, and temporary shocks that recover instantaneously have zero impact on this 
economy. By contrast, temporary shocks may have lasting impact in the vertical economy of panel (b), with the network diagram reproduced below, along with input-output table of this economy. Sector 1 is the most upstream and sector $N$ is the most downstream.

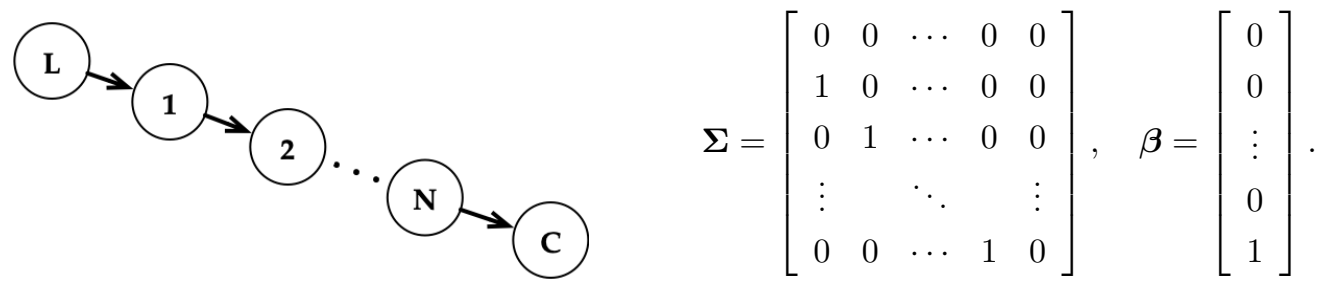

In this vertical economy, each successive power of the input-output matrix contains a smaller identity sub-matrix in the bottom-left and zeros otherwise, and the Leontief-inverse is a lower-triangular matrix of ones:

$$
\boldsymbol{\Sigma}^{s}=\left[\begin{array}{cc}
\boldsymbol{0}_{s \times(N-s)} & \mathbf{0}_{s \times s} \\
\boldsymbol{I}_{(N-s) \times(N-s)} & \mathbf{0}_{(N-s) \times s}
\end{array}\right], \quad(\boldsymbol{I}-\boldsymbol{\Sigma})^{-1}=\left[\begin{array}{ccccc}
1 & 0 & \cdots & 0 & 0 \\
1 & 1 & \cdots & 0 & 0 \\
1 & 1 & \ddots & 0 & 0 \\
\vdots & 1 & \ddots & 1 & \vdots \\
1 & 1 & \cdots & 1 & 1
\end{array}\right] .
$$

For example, when $N=4$,

$$
\boldsymbol{\Sigma}=\left[\begin{array}{llll}
0 & 0 & 0 & 0 \\
1 & 0 & 0 & 0 \\
0 & 1 & 0 & 0 \\
0 & 0 & 1 & 0
\end{array}\right], \quad \boldsymbol{\Sigma}^{2}=\left[\begin{array}{cccc}
0 & 0 & 0 & 0 \\
0 & 0 & 0 & 0 \\
1 & 0 & 0 & 0 \\
0 & 1 & 0 & 0
\end{array}\right], \quad \boldsymbol{\Sigma}^{3}=\left[\begin{array}{cccc}
0 & 0 & 0 & 0 \\
0 & 0 & 0 & 0 \\
0 & 0 & 0 & 0 \\
1 & 0 & 0 & 0
\end{array}\right], \quad(\boldsymbol{I}-\boldsymbol{\Sigma})^{-1}=\left[\begin{array}{cccc}
0 & 0 & 0 & 0 \\
1 & 0 & 0 & 0 \\
1 & 1 & 0 & 0 \\
1 & 1 & 1 & 0
\end{array}\right]
$$

By construction, the Domar weight is identically one for all sectors, $\boldsymbol{\gamma}^{\prime} \equiv \boldsymbol{\beta}^{\prime}(\boldsymbol{I}-\boldsymbol{\Sigma})^{-1}=$ $\mathbf{1}^{\prime}$. TFP shocks in every sector has identical impact on GDP in a static model. In our dynamic economy, however, the welfare impact of sectoral shocks is no longer constant; in fact, the impact of temporary shocks follow

$$
\boldsymbol{v}^{\prime}=\rho \delta\left[1-(1+\rho \delta)^{-N}, \cdots, 1-\left(\frac{1}{1+\rho \delta}\right)^{2}, 1-\frac{1}{1+\rho \delta}, \quad 0\right]
$$

Hence, temporary shocks to sector $i$ are more damaging than to sector $j>i$, despite all sectors having the same Domar weight.

Figure 2 shows the path of GDP over time when each sector in the vertical economy (with $N=4)$ is affected by a temporary, negative TFP shock. As the figure demonstrates, shocks to relatively upstream sectors have long-lasting effects: the economy takes the longest time 
Figure 2: Time path of GDP losses from sectoral shocks in the vertical economy

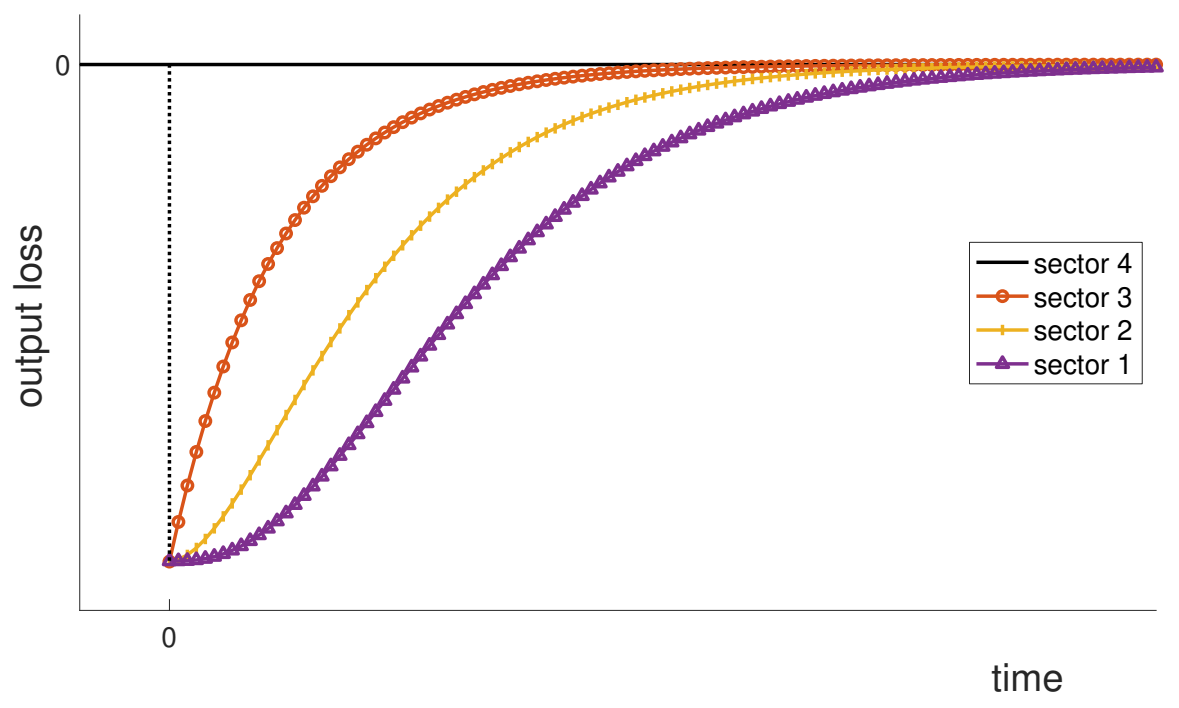

to recover from shocks to sector 1 - the most upstream - and recovers instantaneously from shocks to sector 4 . Consequently, $v_{1}>v_{2}>v_{3}>v_{4}$ as the measure $\boldsymbol{v}^{\prime}$ integrates the entire path of output losses, discounting the future at rate $\rho$.

Connection to Katz Centrality and Upstreamness Temporary shocks are damaging if they affect the sectors with significant sales through distant linkages to the consumer. We now show $v_{i}$ is the product between sector $i$ 's Domar weight and its Katz (1953) centrality of the input-output revenue share matrix. In our context, Katz centrality is a measure of upstreamness: it captures the network-adjusted distance of sectoral supply to the final consumer. Hence, temporary shocks are more damaging to the economy if they affect large sectors that are also upstream and supply disproportionate fractions of outputs to other upstream producers.

Let $\eta_{i} \equiv v_{i} / \gamma_{i}$ be the welfare impact of a temporary shock to sector $i$ relative to the sectoral size. Let $\Theta$ be the input-output revenue share matrix, whose in-th entry is $\theta_{i n} \equiv \sigma_{n i} \gamma_{n} / \gamma_{i}$, i.e. the fraction of revenue that sector $i$ derives from selling to sector $n$. Intuitively, the entries of the expenditure share matrix $\Sigma$ are obtained by dividing the value of intermediate inputs by the sales of the buyer, whereas entries of $\boldsymbol{\Theta}$ are obtained by dividing the value of inputs by the sales of the supplier.

\section{Proposition 4. Welfare impact is the Domar weight times the Katz centrality.}

$$
\boldsymbol{\eta}=\delta\left[\sum_{s=1}^{\infty}\left(\frac{1}{1+\rho \delta} \boldsymbol{\Theta}\right)^{s}\right] \mathbf{1}
$$


Proof. Let $r \equiv \frac{1}{1+\rho \delta}$ and $\boldsymbol{x}^{\prime} \equiv \boldsymbol{\beta}^{\prime}(\boldsymbol{I}-r \boldsymbol{\Sigma})^{-1}$. We have

$$
\begin{gathered}
\rho \eta_{j}=1-x_{j} / \gamma_{j}=1-\beta_{j} / \gamma_{j}-r \sum_{i} \sigma_{i j} x_{i} / \gamma_{j}=\sum_{i} \theta_{j i}-r \sum_{i} \theta_{j i}\left(1-\rho \eta_{i}\right) \\
\Longrightarrow \boldsymbol{\eta}=\delta r \boldsymbol{\Theta}+r \boldsymbol{\Theta}=\delta(\boldsymbol{I}-r \boldsymbol{\Theta})^{-1} r \boldsymbol{\Theta} \mathbf{1}=\delta\left[\sum_{s=1}^{\infty}\left(\frac{1}{1+\rho \delta} \boldsymbol{\Theta}\right)^{s}\right] \mathbf{1} .
\end{gathered}
$$

Katz centrality can be re-written implicitly as $\boldsymbol{\eta}=\frac{\delta}{1+\rho \delta} \boldsymbol{\Theta} \mathbf{1}+\frac{1}{1+\rho \delta} \boldsymbol{\Theta} \boldsymbol{\eta}$, or, in scalar form,

$$
\eta_{i}=\frac{\delta}{1+\rho \delta} \sum_{n=1}^{N} \Theta_{i n}+\frac{1}{1+\rho \delta} \sum_{n=1}^{N} \Theta_{i n} \eta_{n}
$$

The first term on the right-hand-side is a constant $\left(\frac{\delta}{1+\rho \delta}\right)$ times the total fraction of sector $i$ 's output supplied to intermediate producers (rather than the consumer). The second term is the average Katz centrality of the producers that use good $i$ as inputs, weighted by the fraction of $i$ 's output sold to each buyer, and scaled down by a factor $\frac{1}{1+\rho \delta}$. Hence, a sector is Katz-central if it supplies a disproportionate fraction of output to other Katz-central producers.

Katz centrality is a natural notion of upstreamness. ${ }^{3}$ Recall from (4) that the sectoral Domar weight can be written as $\boldsymbol{\gamma}^{\prime}=\boldsymbol{\beta}^{\prime} \sum_{s=0}^{\infty} \boldsymbol{\Sigma}^{s}$, where the $i$-th component of $\boldsymbol{\beta}^{\prime} \boldsymbol{\Sigma}^{s}$ captures the sales of sector $i$ (relative to GDP) that reaches the final consumer through $s$-rounds of input-output linkages. Antràs et al. (2012) defines an upstreamness measure that captures the average number of rounds it takes for sectoral output to reach the final consumer:

$$
\mathrm{Up}_{i}=1 \cdot \frac{\beta_{i}}{\gamma_{i}}+2 \cdot \frac{\left[\boldsymbol{\beta}^{\prime} \boldsymbol{\Sigma}\right]_{i}}{\gamma_{i}}+3 \cdot \frac{\left[\boldsymbol{\beta}^{\prime} \boldsymbol{\Sigma}^{2}\right]_{i}}{\gamma_{i}}+\cdots=\sum_{s=0}^{\infty} \frac{a_{s} \cdot\left[\boldsymbol{\beta}^{\prime} \boldsymbol{\Sigma}^{s}\right]_{i}}{\gamma_{i}}, \quad \text { with } a_{s}=s+1
$$

More generally, $\sum_{s=0}^{\infty} \frac{a_{s} \cdot\left[\boldsymbol{\beta}^{\prime} \boldsymbol{\Sigma}^{s}\right]_{i}}{\gamma_{i}}$ is a measure of sector $i$ 's upstreamness for any increasing and convergent sequence $\left\{a_{s}\right\}_{s=0}^{\infty}$ because such a sequence up-weights sectoral sales that are more distant to the consumer. Katz centrality can also be written in this form using the sequence $a_{s}=\rho^{-1}\left(1-(1+\rho \delta)^{-s}\right)$.

\footnotetext{
${ }^{3}$ The Katz centrality is also isomorphic to the distortion centrality of Liu (2019) in a production network with constant market imperfection wedges.
} 


\section{Eigendecomposition of the Input-Output Matrix and the Dynamical System}

Temporary shocks to large and upstream sectors are disproportionately damaging because input-output linkages are slow to recover from these shocks. We now examine determinants of the welfare measure $\boldsymbol{v}^{\prime}$ from the spectral point of view.

Consider the diagonalization of the input-output table, $\boldsymbol{\Sigma}=\boldsymbol{U} \boldsymbol{\Lambda} \boldsymbol{W}$, where $\boldsymbol{\Lambda}$ is a diagonal matrix of eigenvalues $\left\{\lambda_{k}\right\}_{k=1}^{N}$, and $\boldsymbol{W}=\boldsymbol{U}^{-1}$. The columns of $\boldsymbol{U}$ are the right-eigenvectors, and the rows of $\boldsymbol{W}$ are the corresponding left-eigenvectors. Assuming $\boldsymbol{\Sigma}$ is full-rank, $\boldsymbol{U}$ and $\boldsymbol{W}$ both span the $N$-dimensional complex coordinate space $\mathbb{C}^{N}$ and are therefore are both bases of the space. Without loss of generality, we arrange the eigenvalues and eigenvectors in decreasing absolute value, with $\left|\lambda_{1}\right| \geq\left|\lambda_{2}\right| \geq \cdots \geq\left|\lambda_{N}\right|$. Note that since $\Sigma$ is row-substochastic (i.e., the sum of intermediate expenditure shares must be $\leq 1$ in all sectors, with strict inequality for at least some sectors), the dominant eigenvalue must have absolute value below one, i.e., $\left|\lambda_{1}\right|<1$.

Right Eigenvectors. Let $\boldsymbol{u}_{k}$ denote the $k$-th right-eigenvector, i.e., the $k$-th column of the matrix $\boldsymbol{U}$. This is the vector that, when being multiplied by $\boldsymbol{\Sigma}$ on the left, becomes a scaled version of itself:

$$
\boldsymbol{\Sigma}^{s} \boldsymbol{u}_{k}=\lambda_{k}^{s} \boldsymbol{u}_{k} \quad \text { for all } s \in \mathbb{Z}_{\geq 0}
$$

Now consider a negative TFP shock vector that equals to $\left(-\boldsymbol{u}_{k}\right)$, and suppose $\boldsymbol{u}_{k} \in \mathbb{R}^{N}$. The first round effect lowers sectoral output by $\left(-\boldsymbol{u}_{k}\right)$; the second round effect lowers sectoral output by $\left(-\boldsymbol{\Sigma} \boldsymbol{u}_{k}=-\lambda_{k} \boldsymbol{u}_{k}\right)$; the third round effect lowers sectoral output by $\left(-\lambda_{k}^{2} \boldsymbol{u}_{k}\right)$, and so on. That is, at each round of propagation, the productivity shock vector $\left(-\boldsymbol{u}_{k}\right)$ always reduces sectoral output in proportion to $-\boldsymbol{u}_{k}$, with effects scaled-down by a factor equal to the eigenvalue $\lambda_{k}$ relative to the previous round. In other words, $\boldsymbol{u}_{k}$ is the profile of TFP shocks with every round of general equilibrium effect always in proportion to the first round but decays at rate $\lambda_{k}$ after each round of propagation. We construct the right-eigenvectors so that 2 -norm of $\boldsymbol{u}_{k}$ is equal to 1 for all $k$.

Complex Eigenvalues. In general, the eigenvalues and eigenvectors can be complexvalued. Complex eigenvalues exist in conjugate pairs: when an eigenvalue $\lambda_{k}$ is complex - in which case $\boldsymbol{u}_{k}$ must be complex too - the conjugate transpose $\overline{\lambda_{k}}$ is also an eigenvalue, and the corresponding eigenvector is $\overline{\boldsymbol{u}_{k}}$. Now consider a shock profile $\tilde{\boldsymbol{z}} \equiv \operatorname{Re}\left(\boldsymbol{u}_{k}\right)$. Note 
$\operatorname{Re}\left(\boldsymbol{u}_{k}\right)=\frac{\boldsymbol{u}_{k}+\overline{\boldsymbol{u}_{k}}}{2}$, and following equation must be satisfied:

$$
\Sigma^{s} \operatorname{Re}\left(\boldsymbol{u}_{k}\right)=\operatorname{Re}\left(\lambda_{k}^{s} \boldsymbol{u}_{k}\right)
$$

where the operator $\operatorname{Re}(\cdot)$ selects the real part of a complex vector. Note that, when $\lambda_{k}$ is complex, $\operatorname{Re}\left(\lambda_{k}^{s} \boldsymbol{u}_{k}\right) \neq \operatorname{Re}\left(\lambda_{k}\right) \cdot \operatorname{Re}\left(\lambda_{k}^{s-1} \boldsymbol{u}_{k}\right)$; hence, the shock vector $\operatorname{Re}\left(\boldsymbol{u}_{k}\right)$ is not a scaleddown version of itself when left-multiplied by the input-output table $\boldsymbol{\Sigma}$. The higher rounds of network effects from the shock vector $\operatorname{Re}\left(\boldsymbol{u}_{k}\right)$ no longer decay to zero at a constant rate $\operatorname{Re}\left(\lambda_{k}\right)$; instead, complex eigenvalues introduce oscillatory motion in the impact of negative shocks as the network effects converge to zero under higher and higher rounds. In other words, if we project the network effects $\boldsymbol{\Sigma}^{s} \tilde{\boldsymbol{z}}$ onto an $N$-dimensional vector space, the higher rounds of network effects (higher $s$ ) associated with a real eigenvector shock profile converge to zero following a straight line connecting $\boldsymbol{u}_{k}$ and the origin. In contrast, the network effects of a shock profile $\operatorname{Re}\left(\boldsymbol{u}_{k}\right)$ with a complex eigenvalue would converge to zero following an elliptical spiral.

For expositional purposes, we focus on real-valued eigenvalue and eigenvectors. As we show below, the largest (hence, as we show below, more important) eigenvalues of the realworld input-output tables are all real. Moreover, the imaginary components of any complex eigenvalues are overall significantly smaller than the real components, implying that oscillatory higher-order network effects are small relative to the effects that decays exponentially.

Left Eigenvectors. Let $\boldsymbol{w}_{k}^{\prime}$ denote the $k$-th left-eigenvector, i.e., the $k$-th row of the matrix $\boldsymbol{W} . \boldsymbol{W}$ is the matrix that projects sectoral shocks onto the right-eigenspace. Specifically, any TFP shock vector $\tilde{\boldsymbol{z}}$ can be written as a linear combination $\left\{a_{k}\right\}_{k=1}^{N}$ of the right-eigenvectors

$$
\tilde{\boldsymbol{z}}=\sum_{k=1}^{N} a_{k} \boldsymbol{u}_{k}
$$

or, in matrix notation,

$$
\tilde{\boldsymbol{z}}=\boldsymbol{U} \boldsymbol{a}^{\prime}
$$

The vector $\boldsymbol{a}^{\prime}$ can be obtained by

$$
\boldsymbol{W} \tilde{\boldsymbol{z}}=\boldsymbol{W} \boldsymbol{U} \boldsymbol{a}^{\prime}=\boldsymbol{U}^{-1} \boldsymbol{U} \boldsymbol{a}^{\prime}=\boldsymbol{a}^{\prime}
$$

That is, $a_{k}=\boldsymbol{w}_{k}^{\prime} \tilde{\boldsymbol{z}}$, or in vector form, $\boldsymbol{a}^{\prime}=\boldsymbol{W} \tilde{\boldsymbol{z}}$.

To summarize, the right-eigenvectors capture the shock profiles whose general equilibrium impact decays at rate governed by the corresponding eigenvalues; the left-eigenvectors 
convert shock profiles into coordinates in the right-eigenspace. We sometimes refer to the right-eigenvectors simply as eigenvectors.

Eigen-Decomposition of Domar Weights and Welfare Impact We now use the bases $\boldsymbol{U}$ and $\boldsymbol{W}$ to further decompose the aggregate impact of sectoral shocks.

Proposition 5. Eigen-Decomposition of Domar Weights and Welfare Impact. The Domar weight can be written as

$$
\boldsymbol{\gamma}^{\prime}=\boldsymbol{\beta}^{\prime} \sum_{k=1}^{N} \frac{1}{1-\lambda_{k}} \boldsymbol{u}_{k} \boldsymbol{w}_{k}^{\prime}
$$

The vector $\boldsymbol{v}^{\prime}$ can be written as

$$
\boldsymbol{v}^{\prime}=\delta \boldsymbol{\beta}^{\prime} \sum_{k=1}^{N} \frac{\lambda_{k}}{\left(1-\lambda_{k}\right)\left(1+\rho \delta-\lambda_{k}\right)} \boldsymbol{u}_{k} \boldsymbol{w}_{k}^{\prime} .
$$

Proof. Consider the Domar weight

$$
\begin{aligned}
\boldsymbol{\gamma}^{\prime} & =\boldsymbol{\beta}^{\prime}\left(\sum_{s=0}^{\infty} \boldsymbol{\Sigma}^{s}\right)=\boldsymbol{\beta}^{\prime} \boldsymbol{U}\left(\sum_{s=0}^{\infty} \boldsymbol{\Lambda}^{s}\right) \boldsymbol{W} \\
& =\boldsymbol{\beta}^{\prime} \sum_{k=1}^{N}\left(\sum_{s=0}^{\infty} \lambda_{k}^{s}\right) \boldsymbol{u}_{k} \boldsymbol{w}_{k}^{\prime}=\boldsymbol{\beta}^{\prime} \sum_{k=1}^{N} \frac{1}{1-\lambda_{k}} \boldsymbol{u}_{k} \boldsymbol{w}_{k}^{\prime}
\end{aligned}
$$

The welfare impact

$$
\begin{aligned}
\boldsymbol{v}^{\prime} & =\frac{1}{\rho} \boldsymbol{\beta}^{\prime} \sum_{s=0}^{\infty}\left(1-(1+\rho \delta)^{-s}\right) \boldsymbol{\Sigma}^{s} \\
& =\frac{1}{\rho} \boldsymbol{\beta}^{\prime} \sum_{k=1}^{N}\left(\frac{1}{1-\lambda_{k}}-\frac{1}{1-\frac{1}{1+\rho \delta} \lambda_{k}}\right) \boldsymbol{u}_{k} \boldsymbol{w}_{k}^{\prime} \\
& =\delta \boldsymbol{\beta}^{\prime} \sum_{k=1}^{N} \frac{\lambda_{k}}{\left(1-\lambda_{k}\right)\left(1+\rho \delta-\lambda_{k}\right)} \boldsymbol{u}_{k} \boldsymbol{w}_{k}^{\prime} .
\end{aligned}
$$

The proposition turns the infinite-sum-of-power-series representation of $\boldsymbol{\gamma}^{\prime}$ and $\boldsymbol{v}^{\prime}$ in (4) and (3) into finite sums over eigen components. 
To understand the implication of Proposition 5, first consider a TFP shock profile captured by $\tilde{\boldsymbol{z}}=\boldsymbol{u}_{k}$. Note that

$$
\boldsymbol{w}_{\ell}^{\prime} \boldsymbol{u}_{k}= \begin{cases}1 & \text { if } \ell=k \\ 0 & \text { otherwise }\end{cases}
$$

The shock profile $\boldsymbol{u}_{k}$ 's impact in a static model is therefore captured by

$$
\begin{aligned}
\boldsymbol{\gamma}^{\prime} \boldsymbol{u}_{k} & =\boldsymbol{\beta}^{\prime} \sum_{\ell=1}^{N} \frac{1}{1-\lambda_{\ell}} \boldsymbol{u}_{\ell} \boldsymbol{w}_{\ell}^{\prime} \boldsymbol{u}_{k} \\
& =\frac{1}{1-\lambda_{k}} \boldsymbol{\beta}^{\prime} \boldsymbol{u}_{k} .
\end{aligned}
$$

That is, the shock $\boldsymbol{u}_{k}$ affects static consumption only through the $k$-th eigen component, with the direct effect being $\boldsymbol{\beta}^{\prime} \boldsymbol{u}_{k}$, the $s$-th round indirect network effect being $\lambda_{k}^{s} \boldsymbol{\beta}^{\prime} \boldsymbol{u}_{k}$, and a cumulative effect of $\sum_{s=0}^{\infty} \lambda_{k}^{s} \boldsymbol{\beta}^{\prime} \boldsymbol{u}_{k}=\frac{1}{1-\lambda_{k}} \boldsymbol{\beta}^{\prime} \boldsymbol{u}_{k}$.

We now analyze $\left(\boldsymbol{v}^{\prime} \boldsymbol{u}_{k}\right)$, i.e., the welfare impact of the temporary shock vector $\boldsymbol{u}_{k}$ in our dynamic economy. Since the shock vector $\boldsymbol{u}_{k}$ affects consumption at all times only through the $k$-th eigen component, the impact can be re-written as

$$
\boldsymbol{v}^{\prime} \boldsymbol{u}_{k}=\frac{1}{\rho} \boldsymbol{\beta}^{\prime} \boldsymbol{u}_{k}\left(\sum_{s=0}^{\infty}\left(1-(1+\rho \delta)^{-s}\right) \lambda_{k}^{s}\right) .
$$

The additional term $\left(1-(1+\rho \delta)^{-s}\right)$ assigns zero weight to the direct effect of the shock $(s=$ 0 )-because TFP recovers at $t=0$ - and an increasing sequence of weights to higher-order network effects. The cumulative effect is then scaled by $1 / \rho$ to reflect the fact that we have a dynamic economy with consumer discount rate $\rho$. The expression $\rho^{-1}\left(\sum_{s=0}^{\infty}\left(1-(1+\rho \delta)^{-s}\right) \lambda_{k}^{s}\right)$ further simplifies to $\frac{\lambda_{k}}{\left(1-\lambda_{k}\right)\left(1+\rho \delta-\lambda_{k}\right)}$ as the proposition shows.

Any generic TFP shock vector $\tilde{\boldsymbol{z}}$ can be projected onto the right-eigenspace with $\tilde{\boldsymbol{z}}=$ $\sum_{k=1}^{N} \boldsymbol{u}_{k} a_{k}$, and $a_{k} \equiv \boldsymbol{w}_{k}^{\prime} \tilde{\boldsymbol{z}}$ is its $k$-th coordinate after the projection. The overall effect on welfare is $-\boldsymbol{v}^{\prime} \tilde{\boldsymbol{z}}=-\boldsymbol{v}^{\prime} \sum_{k=1}^{N} \boldsymbol{u}_{k} a_{k}$.

As we show below, quantitatively $\boldsymbol{v}^{\prime}$ has a low-dimensional factor representation, where $\boldsymbol{v}^{\prime} \tilde{\boldsymbol{z}}$ can be approximated closely by its projection onto the first $K(K=4)$ eigenvectors:

$$
\boldsymbol{v}^{\prime} \tilde{\boldsymbol{z}} \approx \boldsymbol{v}^{\prime} \sum_{k=1}^{K} \boldsymbol{u}_{k} a_{k}
$$

Proposition 5 shows why this is the case and also why the Domar weight does not have a good approximation in a low-dimensional sub-eigenspace. To see this, consider two distinct 
shock profiles captured by real right-eigenvectors $\boldsymbol{u}_{k}$ and $\boldsymbol{u}_{\ell}$ with $\left|\lambda_{k}\right|<\left|\lambda_{\ell}\right|$. Let $\epsilon \equiv \frac{\left|\boldsymbol{\beta}^{\prime} \boldsymbol{u}_{k}\right|}{\left|\boldsymbol{\beta}^{\prime} \boldsymbol{u}_{\ell}\right|}$ denote the relative loadings of the consumption share vector on these two eigenvectors. The relative impact between the eigenvector shock profiles on aggregate consumption in the static model is

$$
\frac{\left|\gamma^{\prime} \boldsymbol{u}_{k}\right|}{\left|\gamma^{\prime} \boldsymbol{u}_{\ell}\right|}=\frac{1-\lambda_{\ell}}{1-\lambda_{k}} \times \epsilon
$$

On the other hand, their relative impact in our dynamic model is

$$
\frac{\left|\boldsymbol{v}^{\prime} \boldsymbol{u}_{k}\right|}{\left|\boldsymbol{v}^{\prime} \boldsymbol{u}_{\ell}\right|}=\underbrace{\frac{\left|\lambda_{k}\right|\left(1+\rho \delta-\lambda_{\ell}\right)}{\left|\lambda_{\ell}\right|\left(1+\rho \delta-\lambda_{k}\right)}}_{<1} \times \frac{1-\lambda_{\ell}}{1-\lambda_{k}} \times \epsilon
$$

That is, relative to the static model, our dynamic model up-weights the relative importance of right-eigenvectors shock profiles with greater eigenvalues and, conversely, down-weights righteigenvector shock profiles with lower eigenvalues. These difference could be very significant: as we show below, for the U.S. economy, the dominant eigenvalue is $\left|\lambda_{1}\right| \approx 0.54$, and the 100 -th is $\left|\lambda_{100}\right| \approx 0.03$. The relative importance between the 100 -th and the first eigenvector $\boldsymbol{u}_{100}$ and $\boldsymbol{u}_{1}$ in our economy is bounded above by

$$
\frac{\left|\lambda_{100}\right|\left(1+\rho \delta-\lambda_{1}\right)}{\left|\lambda_{1}\right|\left(1+\rho \delta-\lambda_{100}\right)} \times \frac{1-\lambda_{1}}{1-\lambda_{100}} \epsilon \leq \frac{\left|\lambda_{100}\right|}{\left|\lambda_{1}\right|} \times \frac{1-\lambda_{1}}{1-\lambda_{100}} \epsilon=0.0263 \times \epsilon,
$$

whereas their relative importance in the static model is

$$
\frac{1-\lambda_{1}}{1-\lambda_{100}} \epsilon=0.47 \times \epsilon
$$

Despite the small eigenvalue $\left|\lambda_{100}\right|$, the 100-th eigenvector is still $47 \epsilon \%$ as important as the dominant eigenvector in the static model; by contrast, $\boldsymbol{u}_{100}$ is at most $2.6 \epsilon \%$ as important as $\boldsymbol{u}_{1}$ in our dynamic model. The upper-bound $2.6 \epsilon \%$ is achieved under the limit $\rho \delta \rightarrow \infty$, corresponding to the case with infinite discounting or infinite adjustment costs. When $\rho \delta$ is finite, the 100-th eigenvector becomes even less important relative to the dominant eigenvector; for instance, when $\rho=\delta=10 \%$, their relative importance is given by $\frac{\left|\lambda_{100}\right|\left(1.01-\lambda_{1}\right)}{\left|\lambda_{1}\right|\left(1.01-\lambda_{1}\right)} \times \frac{1-\lambda_{1}}{1-\lambda_{100}} \epsilon=0.0126 \times \epsilon$.

Intuitively, eigenvectors with large eigenvalues represent shock profiles that decay slowly; these shock profiles generate disproportionately high impact via higher rounds of network effects. The fact that Domar weights do not discount the direct and initial rounds of network effects imply that even eigenvectors with small eigenvalues may have a sizable contribution in explaining TFP shocks in the static model. Sectoral shocks in the static model therefore do not have a low-dimensional representation. By contrast, because dynamic adjustment 
costs significantly down-weight the direct and initial rounds of network effects, $\boldsymbol{v}^{\prime}$ may have a factor representation as long as $\left|\lambda_{k}\right|$ declines relatively fast in $k$.

\section{Factor Structure of the U.S. Input-Output Table}

We now turn to the 2012 U.S. input-output table published by the U.S. Bureau of Labor Statistics. We show that the high-dimensional input-output table-171 by 171 sectors under broad categories of agriculture, mining, manufacturing, and services ${ }^{4}$ - has a lowdimensional, 4-factor structure in terms of its susceptibility to temporary shocks: the $\boldsymbol{v}^{\prime}$ vector essentially loads on only four eigenvectors of the $\boldsymbol{\Sigma}$ matrix. These correlated eigenvectors explain $95 \%$ of the variations in $\boldsymbol{v}^{\prime}$ and they jointly capture three clusters of sectors in the economy: 1) the heavy manufacturing sectors including iron, steel, and machineries; 2) light manufacturing sectors of consumer products including food and textiles; and 3) the chemical manufacturing sectors. We show such a factor structure emerges only when assessing the impact of temporary shocks. In contrast, the economy does not have a low-dimensional, factor representation for permanent shocks, as the Domar weights have significant loadings on over 150 eigenvectors. For simplicity, we present our results assuming $\rho=\delta=10 \%$ but this choice is immaterial for the results here. ${ }^{5}$

Table 1: Welfare elasticity to temporary sectoral shocks in the U.S.

\begin{tabular}{ll}
\hline 10 sectors with the highest $v_{i}$ & 10 sectors with the smallest $v_{i}$ \\
\hline Real estate & Community and vocational rehabilitation services \\
Wholesale trade & Gambling industries (except casino hotels) \\
Agencies, brokerages, and other insurance related activities & Other furniture related product manufacturing \\
Oil and gas extraction & Personal care services \\
Basic chemical manufacturing & Amusement parks and arcades \\
Management of companies and enterprises & Grantmaking, giving services, social advocacy organizations \\
Petroleum and coal products manufacturing & Food and beverage stores \\
Advertising, public relations, and related services & Tobacco manufacturing \\
Nonferrous metal (except aluminum) production \& processing & Motor vehicle manufacturing \\
Motor vehicle parts manufacturing & Other transportation equipment manufacturing \\
\hline
\end{tabular}

\footnotetext{
${ }^{4} 13$ sectors from the original 184-by-184 BLS input-output table do not use or supply any intermediate inputs and therefore do not interact with the rest of the network. These sectors are all in services, including offices of dentists, individual family services, home health care services, etc. We drop these sectors when performing the eigendecomposition.

${ }^{5}$ In Appendix A, we show the 4-factor representation is robust under alternative values of $\rho$ and $\delta$. In fact, the first 4 eigenvectors explain over $90 \%$ of the variation in $\boldsymbol{v}^{\prime}$ even in the limit as $\rho \delta \rightarrow \infty$.
} 
Table 1 lists the top-10 most important and least important sectors for the U.S. in terms of $\boldsymbol{v}^{\prime}$, the welfare elasticity to temporary sectoral shocks. As intuitions suggest, the most important ones are large sectors that supply to many other producers. The top-10 list includes very large sectors such as real estate and wholesale trade, whose sales-to-GDP ratios add to $24 \%$. The list also includes much smaller but very upstream manufacturing sectors such as chemical and metal sectors. On the right side of the table, sectors with low welfare impact are those that are small and downstream, including many service sectors.

Figure 3: Decay of eigen components

Sorted by the absolute value of eigenvalues
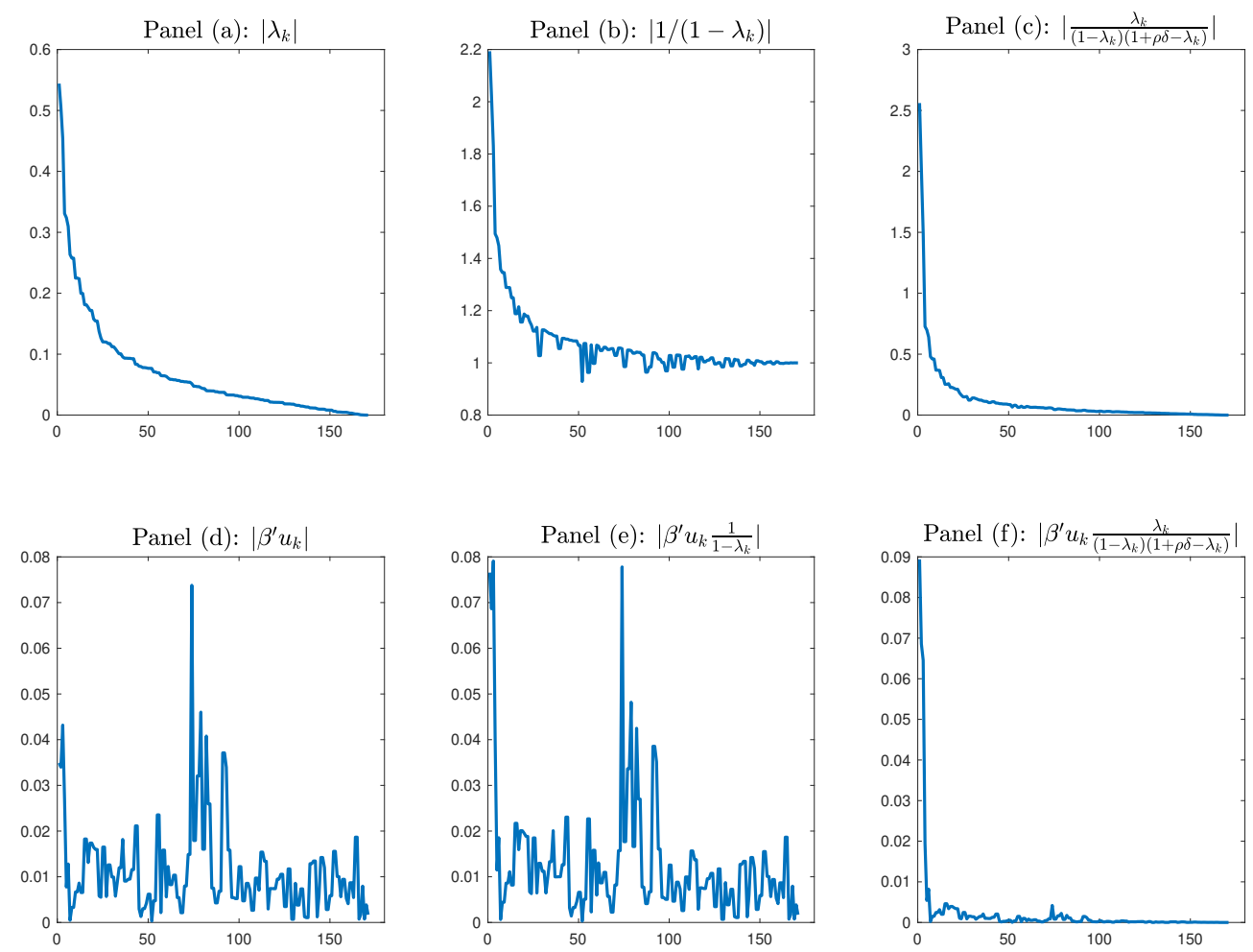

We now describe the first empirical results of the paper: the welfare elasticity to temporary shocks in the U.S. can be well-approximated in a low-dimensional sub-eigenspace.

In Figure 3, building on Proposition 5, the top panels (a)-(c) respectively show the eigenvalues $\left|\lambda_{k}\right|$, their geometric sum $\left|1 /\left(1-\lambda_{k}\right)\right|$, and the term $\left|\frac{\lambda_{k}}{\left(1-\lambda_{k}\right)\left(1+\rho \delta-\lambda_{k}\right)}\right|$ for the U.S. input-output table. The bottom panels (d)-(f) respectively show $\left|\boldsymbol{\beta}^{\prime} \boldsymbol{u}_{k}\right|,\left|\boldsymbol{\beta}^{\prime} \boldsymbol{u}_{k} /\left(1-\lambda_{k}\right)\right|$, and $\left|\boldsymbol{\beta}^{\prime} \boldsymbol{u}_{k} \frac{\lambda_{k}}{\left(1-\lambda_{k}\right)\left(1+\rho \delta-\lambda_{k}\right)}\right|$. In all panels, indices $k$ are arranged in decreasing order of the absolute eigenvalues. 
Panel (a) shows the decay of the eigenvalues of the input-output matrix. The term $\left|1 /\left(1-\lambda_{k}\right)\right|$ shown in panel (b) captures the contribution of the $k$-th eigenvector to the geometric series in the matrix $(\boldsymbol{I}-\boldsymbol{\Sigma})^{-1}$. The fact that $\left|1 /\left(1-\lambda_{k}\right)\right|$ does not converge to zeroit converges to 1 as $\left|\lambda_{k}\right| \rightarrow 0$-implies that the matrix does not have a low-dimensional representation, as even eigenvectors after the 100th may be important. ${ }^{6}$ The term $\left|\frac{\lambda_{k}}{\left(1-\lambda_{k}\right)\left(1+\rho \delta-\lambda_{k}\right)}\right|$ shown in panel (c), on the other hand, exhibit very rapid decay towards zero; this implies that the matrix $(\boldsymbol{I}-\boldsymbol{\Sigma})^{-1}-\left(\boldsymbol{I}-\frac{\boldsymbol{\Sigma}}{1+\rho \delta}\right)^{-1}$ potentially has a low-dimensional representation.

Whether the Domar weight $\gamma^{\prime}=\boldsymbol{\beta}^{\prime}(\boldsymbol{I}-\boldsymbol{\Sigma})^{-1}$ or the welfare elasticity to temporary shocks $\boldsymbol{v}^{\prime}=\frac{1}{\rho} \boldsymbol{\beta}^{\prime}\left[(\boldsymbol{I}-\boldsymbol{\Sigma})^{-1}-\left(\boldsymbol{I}-\frac{\boldsymbol{\Sigma}}{1+\rho \delta}\right)^{-1}\right]$ can be well-approximated in a low-dimensional sub-eigenspace depends also on the loading of the consumption vector $\boldsymbol{\beta}^{\prime}$ on each eigenvector. The loadings $\left|\boldsymbol{\beta}^{\prime} \boldsymbol{u}_{k}\right|$ are shown in panel (d), and the contribution of each $k$-th eigenvector to the Domar weight is shown in panel (e). As the figures shows, the consumption vector has significant loadings on many eigenvectors, and so does the Domar weight. For instance, both panels (d) and (e) show spikes around the group of eigenvectors indexed around 75 to 80 - capturing sectors related to healthcare - and around 81 to 85-capturing sectors related to automobiles. These eigenvectors have low eigenvalues, evidenced from panel (a), but they are nevertheless very important for the Domar weight because the consumer expenditure share $\boldsymbol{\beta}^{\prime}$ loads significantly on these eigenvectors, meaning the consumption expenditure shares on healthcare and automobiles are high. Jointly, the two "private hospitals" and "motor vehicle manufacturing" sectors account for over $10 \%$ of the final consumption share. This is even before accounting for other related but smaller sectors such as "medical equipment and supplies manufacturing", "medical and diagnostic laboratories", "other ambulatory health care services", "motor vehicle body and trailer manufacturing", "motor vehicle parts manufacturing", and "motor vehicle and parts dealers".

We now turn to the analysis of the welfare elasticity to temporary shocks, $\boldsymbol{v}^{\prime}$. Panel (f) stands in sharp contrast with panel (e) and shows that only the initial few eigenvectors are important in explaining variations in $\boldsymbol{v}^{\prime}$; that is, the impact of any temporary TFP shock vector $\tilde{\boldsymbol{z}}$ can be well-approximated by the projection of the shock onto a low-dimensional sub-eigenspace spanned by the first few eigenvectors. As the discussion following Proposition 5 shows this is precisely due to the rapid decay of $\left|\frac{\lambda_{k}}{\left(1-\lambda_{k}\right)\left(1+\rho \delta-\lambda_{k}\right)}\right|$ towards zero as shown in panel (c). Even though $\boldsymbol{\beta}^{\prime}$ loads significantly onto some of the high-indexed eigenvectors, the sectors underlying these eigenvectors (e.g. hospitals and automobiles) are very downstream, meaning they mostly supply directly to the final consumer and do not supply strongly to other intermediate sectors. Consequently, these eigenvectors become unimportant in explaining the

\footnotetext{
${ }^{6}$ Certain entries in panel (b) are below one because some of the eigenvalues are negative.
} 
variation in $\boldsymbol{v}^{\prime}$.

Figure 4 reproduces panels (d)-(f) of figure 3 by re-ordering the objects shown in each panel according to declining absolute values (e.g., objects in panel (d) is sorted in declining order of $\left|\boldsymbol{\beta}^{\prime} \boldsymbol{u}_{k}\right|$ rather than declining $\left.\left|\lambda_{k}\right|\right)$. Figure 4 confirms the message in figure 3 even after sorting: the importance of eigen component exhibits very rapid decay in panel (f) are close to zero after the few initial components; by contrast, a large number of components remain important in panels (d) and (e).

\section{Figure 4: Decay of eigen components}

Sorted by the absolute value of objects in each panel
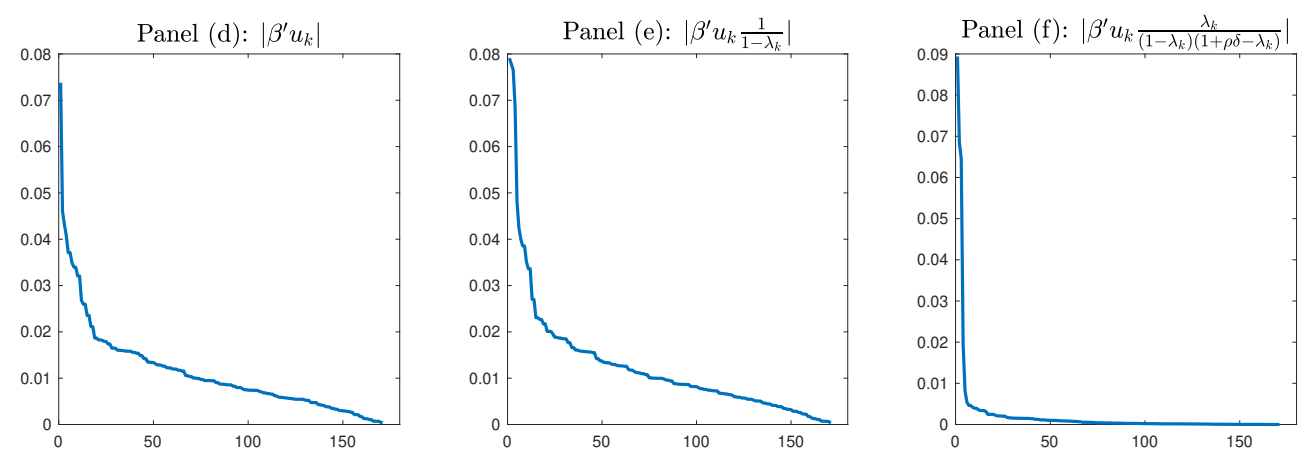

How many eigenvectors are needed to approximate $\boldsymbol{v}^{\prime}$ ? Let $\boldsymbol{g}_{k}^{\prime} \equiv \frac{\lambda_{k}}{\left(1-\lambda_{k}\right)\left(1+\rho \delta-\lambda_{k}\right)} \boldsymbol{\beta}^{\prime} \boldsymbol{u}_{k} \boldsymbol{w}_{k}^{\prime}$ be the $k$-th eigen component of $\boldsymbol{v}^{\prime}$, and let $\boldsymbol{v}_{(h)}^{\prime} \equiv \delta \sum_{k=1}^{h} \boldsymbol{g}_{k}$ denote the partial sum of the first $h$ eigen components. Note that $\boldsymbol{v}^{\prime}=\boldsymbol{v}_{(N)}^{\prime}$.

Figure 5 scatter plots $\boldsymbol{v}_{(h)}^{\prime}$ against $\boldsymbol{v}^{\prime}$ for $h \leq 6$; the red line in each panel is the 45-degree line which indicates that $\boldsymbol{v}_{(h)}$ is close to $\boldsymbol{v}$. As the figure shows, $\boldsymbol{v}_{(4)}^{\prime}$ approximates $\boldsymbol{v}^{\prime}$ very well, and additional 5th and 6th eigen components do not seem to significantly improve the fit. 
Figure 5: Welfare impact from the initial eigenvectors $\left(\boldsymbol{v}_{(h)}^{\prime}\right)$ plotted against $\boldsymbol{v}^{\prime}$
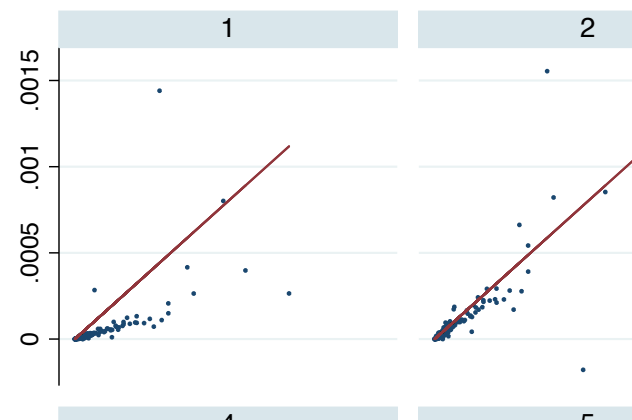

\begin{tabular}{l|l}
2 & 3
\end{tabular}
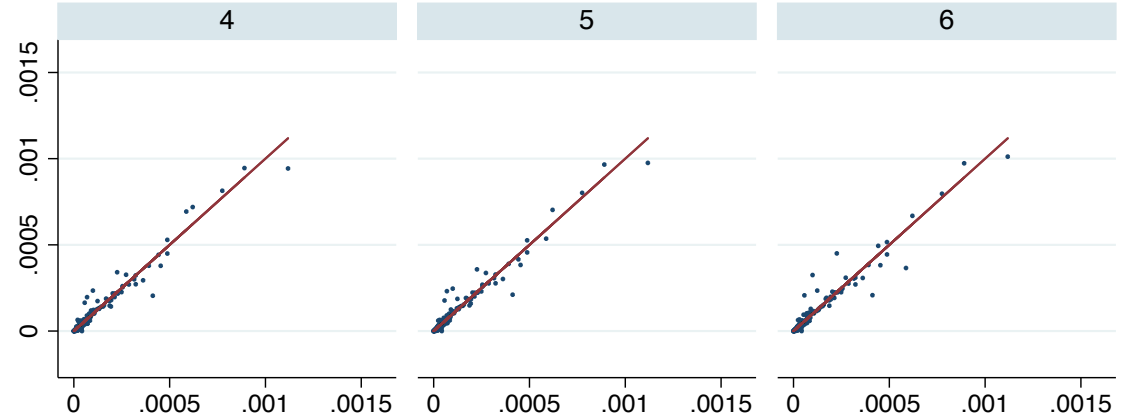

Table 2 shows the regression of $\boldsymbol{v}_{(h)}^{\prime}$ on $\boldsymbol{v}^{\prime}$ for $h \in\{1, \ldots, 6\}$ and reports the slope coefficients and adjusted $R^{2}$. The results show that the first 3 eigenvectors capture $76 \%$ of the variation in $\boldsymbol{v}^{\prime}$; the first 4 eigenvectors capture $95 \%$ of the variation. That is, most of the welfare impact of any sectoral shock $\tilde{\boldsymbol{z}}$ can by explained by the loading of the shock on the first four eigenvectors.

Table 2: Regression of $\boldsymbol{v}_{(h)}^{\prime}$ on $\boldsymbol{v}^{\prime}$

\begin{tabular}{ccccccc}
\hline$h$ & 1 & 2 & 3 & 4 & 5 & 6 \\
\hline slope & 0.53 & 0.82 & 1.01 & 0.97 & 0.97 & 0.96 \\
\hline$R^{2}$ & 0.39 & 0.58 & 0.76 & 0.95 & 0.96 & 0.94 \\
\hline
\end{tabular}

Which sectors do the first four eigenvectors represent? Recall that each eigenvector $\boldsymbol{u}_{k}$ represents a TFP shock profile, under which the network effects decay at exponentially at rate $\lambda_{k}$ and that the cumulative welfare impact is $-\boldsymbol{v}^{\prime} \boldsymbol{u}_{k}=-\frac{\lambda_{k}}{\left(1-\lambda_{k}\right)\left(1+\rho \delta-\lambda_{k}\right)} \boldsymbol{\beta}^{\prime} \boldsymbol{u}_{k}$. 
Figure 6: The first four eigenvectors of $\Sigma$

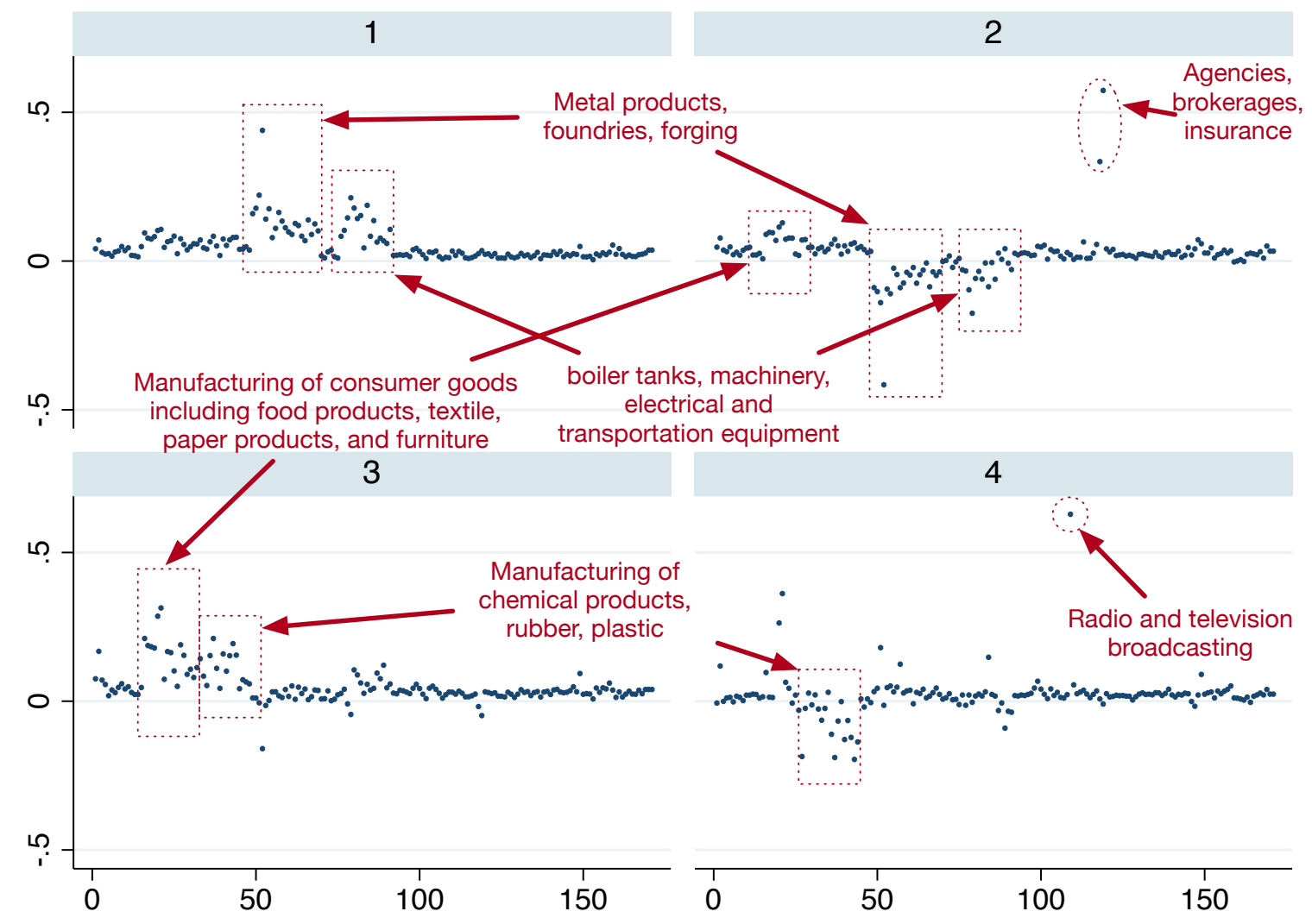

Figure 6 visualizes the first four eigenvectors. The $\mathrm{X}$-axis represent the sectoral ordering according to the BLS input-output table, which roughly arranges broad sector groups by agriculture, food manufacturing, chemical products, metals, heavy manufacturing, and services. In the figure, we indicate the broad groups of sectors that these eigenvectors represent; Tables 4 and 5 provide more detailed lists of sector names. Table 3 lists the first four eigenvalues and the loading of the consumption vector on the corresponding eigenvectors. Because input-output tables are not symmetric, the eigenvectors are not orthogonal to each other. In fact, many eigenvectors are correlated, thereby picking up shocks to the same groups of sectors.

Table 3: Eigenvalues and the consumption loadings of the first four eigenvector

\begin{tabular}{ccccc}
\hline$k$ & 1 & 2 & 3 & 4 \\
\hline$\lambda_{k}$ & 0.544 & 0.505 & 0.454 & 0.331 \\
\hline $\boldsymbol{\beta}^{\prime} \boldsymbol{u}_{k}$ & 0.035 & 0.034 & 0.043 & 0.027 \\
\hline
\end{tabular}


The first eigenvector $\boldsymbol{u}_{1}$ represents shocks to the heavy manufacturing sectors, including metal products, foundries, forging and stamping, and as well as the production of boiler tanks, machinery, electrical and transportation equipment. This eigenvector captures the vector of TFP shocks under which the economic damage to GDP lasts the longest time after TFP recovers.

The second eigenvector $\boldsymbol{u}_{2}$ very strongly and negatively correlates with the first, with Pearson correlation coefficient of -0.59 between $\boldsymbol{u}_{1}$ and $\boldsymbol{u}_{2}$. The eigenvector $\boldsymbol{u}_{2}$ activates three groups of industries. First and most notably, $\boldsymbol{u}_{2}$ has large positive entries for the two sectors relating to agencies, brokerages, and insurance. Second, $\boldsymbol{u}_{2}$ has positive entries for the manufacturing of consumer goods including food, textile, paper products, and furniture. Third, $\boldsymbol{u}_{2}$ has negative entries on the heavy manufacturing industries, partly neutralizing the shock profile from the first eigenvector.

The third eigenvector $\boldsymbol{u}_{3}$ correlates positively with $\boldsymbol{u}_{2}$ - correlation coefficient 0.36 -by having positive entries on the manufacturing of consumer goods. In addition, $\boldsymbol{u}_{3}$ also includes sectors that manufacture chemicals, plastic, and rubber products.

The fourth eigenvector has close-to-zero correlations with the previous three eigenvectors. The new sector picked up by $\boldsymbol{u}_{4}$ is radio and television broadcasting; in addition, $\boldsymbol{u}_{4}$ also has negative entries on the manufacturing of chemicals, plastic, and rubber products, partly neutralizing the shock profiles represented by $\boldsymbol{u}_{3}$.

Table 4: The 1st \& 2nd eigenvector shock profiles: 10 largest entries by absolute value

\begin{tabular}{|c|c|c|c|}
\hline$\left[\boldsymbol{u}_{1}\right]_{i}$ & & {$\left[\boldsymbol{u}_{2}\right]_{i}$} & \\
\hline $\begin{array}{l}\text { Nonferrous metal (except aluminum) production } \\
\text { and processing }\end{array}$ & 0.439 & $\begin{array}{l}\text { Agencies, brokerages, and other insurance related } \\
\text { activities }\end{array}$ & 0.574 \\
\hline $\begin{array}{l}\text { Alumina and aluminum production and } \\
\text { processing }\end{array}$ & 0.221 & Insurance carriers & 0.334 \\
\hline $\begin{array}{l}\text { Other electrical equipment and component } \\
\text { manufacturing }\end{array}$ & 0.213 & Animal slaughtering and processing & 0.128 \\
\hline Railroad rolling stock manufacturing & 0.187 & Dairy product manufacturing & 0.114 \\
\hline Motor vehicle manufacturing & 0.178 & Electrical equipment manufacturing & -0.097 \\
\hline Steel product manufacturing from purchased steel & 0.178 & Steel product manufacturing from purchased steel & -0.103 \\
\hline Forging and stamping & 0.175 & Forging and stamping & -0.110 \\
\hline $\begin{array}{l}\text { Boiler, tank, and shipping container } \\
\text { manufacturing }\end{array}$ & 0.163 & $\begin{array}{l}\text { Alumina and aluminum production and } \\
\text { processing }\end{array}$ & -0.140 \\
\hline Iron and steel mills and ferroalloy manufacturing & 0.159 & $\begin{array}{l}\text { Other electrical equipment and component } \\
\text { manufacturing }\end{array}$ & -0.176 \\
\hline Motor vehicle parts manufacturing & 0.153 & $\begin{array}{l}\text { Nonferrous metal (except aluminum) production } \\
\text { and processing }\end{array}$ & -0.416 \\
\hline
\end{tabular}


Table 5: The 3rd \& 4th eigenvector shock profiles: 10 largest entries by absolute value

\begin{tabular}{|c|c|c|c|}
\hline$\left[\boldsymbol{u}_{3}\right]_{i}$ & & {$\left[\boldsymbol{u}_{4}\right]_{i}$} & \\
\hline Animal slaughtering and processing & 0.314 & Radio and television broadcasting & 0.628 \\
\hline Dairy product manufacturing & 0.286 & Animal slaughtering and processing & 0.362 \\
\hline Animal food manufacturing & 0.211 & Dairy product manufacturing & 0.263 \\
\hline $\begin{array}{l}\text { Resin, synthetic rubber, and artificial synthetic } \\
\text { fibers and filaments manufacturing }\end{array}$ & 0.211 & $\begin{array}{l}\text { Alumina and aluminum production and } \\
\text { processing }\end{array}$ & 0.180 \\
\hline Plastics product manufacturing & 0.194 & Railroad rolling stock manufacturing & 0.148 \\
\hline Textile mills and textile product mills & 0.190 & Paint, coating, and adhesive manufacturing & -0.129 \\
\hline Grain and oilseed milling & 0.187 & Rubber product manufacturing & -0.137 \\
\hline Sugar and confectionery product manufacturing & 0.183 & Textile mills and textile product mills & -0.186 \\
\hline $\begin{array}{l}\text { Fruit and vegetable preserving and specialty food } \\
\text { manufacturing }\end{array}$ & 0.179 & $\begin{array}{l}\text { Resin, synthetic rubber, and artificial synthetic } \\
\text { fibers and filaments manufacturing }\end{array}$ & -0.189 \\
\hline Animal production and aquaculture & 0.168 & Plastics product manufacturing & -0.196 \\
\hline
\end{tabular}

Altogether, the eigenvectors $\boldsymbol{u}_{1}$ through $\boldsymbol{u}_{4}$ form a 4-dimensional subspace of the 171dimensional vector space in which the U.S. input-output table lies. It may appear puzzling at first that the sectors represented by these four eigenvectors do not seem to coincide with the sectors with high welfare impacts as listed in Table 1. There is no inconsistency: the welfare impact of any temporary TFP shock vector $\tilde{\boldsymbol{z}}$ can be well-approximated by projecting $\tilde{\boldsymbol{z}}$ onto this subspace, $\boldsymbol{v}^{\prime} \tilde{\boldsymbol{z}} \approx \sum_{k=1}^{4} \boldsymbol{v}^{\prime} \boldsymbol{u}_{k} a_{k}$, i.e., approximating $\tilde{\boldsymbol{z}}$ with a linear combinations of $\boldsymbol{u}_{1}$ through $\boldsymbol{u}_{4}$, with coordinates $a_{k}=\boldsymbol{w}_{k}^{\prime} \tilde{\boldsymbol{z}}$ obtained using the corresponding left-eigenvectors. Table 6 shows the 4-dimensional coordinates for shocking each of the 10 sectors with the highest welfare impact individually and no other sectors. As an example, the sector "Agencies, brokerages, and other insurance related activities" has a positive coordinate on $\boldsymbol{u}_{2}$, which picks up shocks to this sector very strongly but also shocks to heavy manufacturing products (negatively) and consumer goods (positively); see Figure 6. To isolate the shock to agencies, brokerages and insurance, the sector loads positively on $\boldsymbol{u}_{1}$ and negatively on $\boldsymbol{u}_{3}$ to neutralize the other sectors picked up by $\boldsymbol{u}_{2}$. 
Table 6: Low dimensional representation of TFP shocks to vulnerable sectors in the U.S.

\begin{tabular}{lcccc}
\hline \multirow{2}{*}{10 sectors with the highest $v_{i}$} & \multicolumn{3}{c}{ Loadings on the first 4 eigenvectors } \\
\cline { 2 - 5 } & 1st & 2nd & 3rd & 4 th \\
\hline Real estate & 0.29 & 0.10 & 0.62 & 1.05 \\
Wholesale trade & 0.44 & 0.02 & 0.70 & 0.39 \\
Agencies, brokerages, and other insurance related activities & 0.89 & 1.54 & -1.50 & -0.38 \\
Oil and gas extraction & 0.29 & 0.03 & 0.86 & -0.64 \\
Basic chemical manufacturing & 0.46 & 0.06 & 1.75 & -4.57 \\
Management of companies and enterprises & 0.17 & 0.03 & 0.37 & 0.20 \\
Petroleum and coal products manufacturing & 0.23 & 0.02 & 0.51 & -0.15 \\
Advertising, public relations, and related services & 0.12 & 0.04 & 0.26 & 0.39 \\
Nonferrous metal (except aluminum) production \& processing & 1.60 & -0.20 & -1.19 & -0.43 \\
Motor vehicle parts manufacturing & 0.08 & 0.01 & 0.15 & 0.15 \\
\hline
\end{tabular}

\section{Leontief Targeting of Nazi Germany and Imperial Japan}

Until now we have interpreted the vector $\boldsymbol{v}^{\prime}$ as the welfare impact of slow recovery in sectoral output from temporary shocks to TFP that recovers instantaneously. An equivalent interpretation of $\boldsymbol{v}^{\prime}$ is the welfare impact if a sector's production were to be halted temporarily and output were to be destroyed.

One of the first applications of input-output analysis developed by Leontief was to establish the priorities in targeting for strategic bombing of the Axis powers, in particular Nazi Germany and Imperial Japan. Guglielmo (2008) describes that Leontief was a part of the Enemy Objectives Unit (EOU), a group of economists tasked with the analysis of targeting:

"The economists ... had a comparative advantage in answering the ... question lof How great is the impairment to the enemy's war efforts per unit of destruction], which required familiarity with the enemy's industrial sector and the inputs required types of output. This question could be quite complicated as a result of the interconnectedness of the component sectors ... This insight, which became known as input-output analysis would result in a Nobel Prize in economics."

One important concept was that of depth, a measure of how long it took for damage to have an impact on enemy capacity on the battlefield. The final products such as tanks had less 
depth compared to the intermediate products.

The EOU memoranda compiled by Rostow (1981) discuss the criteria for target selection by the EOU economists. For instance, Salant (1942) wrote in one memorandum:

"it is better to attack a factory the loss of whose output will have widespread effect in causing stoppages elsewhere than one which is a relatively isolated unit in the industrial system."

The E.O.U. Special Report No.9 (1943) also stated

"the most attractive target groups, for general attack on war production are... in the range of components: bearings, the Bosch line, tires, and the other familiar items. It is clear that a time interval will elapse..."

Harrison (2020) summarizes the strategy of economic warfare by the Allies as indirectly attacking the enemy through its supply chain. Bollard (2020) further argued that

"Leontief's input-output provided an economic mapping that the generals could readily understand."

There are also several criticisms of this input-output strategy. First, the United States Strategic Bombing Survey conducted after the war to assess the effectiveness of strategic bombing concluded that bombing had a limited impact on the Nazi economy (Guglielmo (2008)). Similarly, the input-output models assumed the fixed coefficients while in reality the Nazi economy was able to substitute to, perhaps less efficient, but still workable alternatives (Olson (1962), Harrison (2020)).

While, of course, there were many other reasons for target selection such as political and military aims, our model is a modern version of the analysis of the EOU economists fighting against the Axis. In our setup, the welfare impact $v_{i}$ can be seen as the aggregate economic impact of damaging sector $i$ in Nazi Germany and Imperial Japan. In what follows, we estimate the economic impact of shocking each sector for these two countries before the World War II. More broadly, we use this section to also illustrate a variety of other features of our model such as the cross-sectoral impact of shocks over several time horizons.

Specifically, we digitize the 40-by-40 industries input-output table of Germany in 1936 from Fremdling and Staeglin (01 Nov. 2014), and we translate and digitize the 23-by23 industries input-output table of pre-war Japan in 1935 from Nishikawa and Koshihara (1981). ${ }^{7}$ Of course, these data sources were not available to EOU economists at the time and

\footnotetext{
${ }^{7}$ Because the input-output table of Japan in 1935 is not available from digital sources, we provide our digitized version in Appendix B Table 11.
} 
they almost surely did not fully represent the mobilized war time economy, but it is useful to analyze them as they are the best available current sources of information.

First, we provide the list of sectors to which temporary shocks generate the largest impact. Second, we show, for the purpose of finding vulnerability to temporary shocks, both of these input-output tables also exhibit low-dimensional representations: the first three eigenvectors explain $92 \%$ of the variation in $\boldsymbol{v}^{\prime}$ for Imperial Japan and $85 \%$ for Nazi Germany. Third, we provide an interpretation of the first three eigenvectors for both economies. Fourth, we demonstrate the over-time impact of shocks to each sector on every other sector of the economy, and we show shocks to the metal sectors tend to have lasting damage across both for the pre-WWII Germany and Japan.

Table 7 shows the top- 5 most important sectors in terms of $\boldsymbol{v}^{\prime}$, the welfare elasticity to temporary shocks, for Germany in 1936 (left panel) and Japan in 1935 (right panel). Iron and steel products, or metals in general, are important for both economies because they are upstream and because shocks to these sectors destroy many network linkages that could take a long time to recover; we provide further evidence below. Textiles and agriculture are also important for both economies because these sectors represent a significant fraction of GDP.

Table 7: Welfare elasticity to temporary sectoral shocks in pre-WWII Germany and Japan

\begin{tabular}{ll}
\hline 5 sectors with the highest $v_{i}$ for Germany in 1936 & 5 sectors with the highest $v_{i}$ for Japan in 1935 \\
\hline Basic iron and steel products & Agriculture, forestry \\
Transport and communication & Metals \\
Other services & Chemicals \\
Textiles & Textile/personal goods \\
Agriculture & Commerce \\
\hline
\end{tabular}

Recall that $\boldsymbol{v}_{(h)}^{\prime}$ is the $h$-dimensional approximation of $\boldsymbol{v}^{\prime}$ based on the first $h$ eigen components. Table 8 shows the regression of $\boldsymbol{v}_{(h)}^{\prime}$ on $\boldsymbol{v}^{\prime}$ for $h \leq 4$ and reports the slope coefficients and adjusted $R^{2}$. The results show that the first 3 eigenvectors capture $85 \%$ and $92 \%$ of the variation respectively for the pre-WWII Germany and Japan, implying that, similar to the modern U.S. economy, most of the welfare impact of any sectoral shock $\tilde{\boldsymbol{z}}$ in these pre-WWII economies can by explained by the loading of the shock on the first three eigenvectors in these economies. As we have explained, such low-dimensional representation does not exist for the Domar weight, as the representation is possible only because $\boldsymbol{v}^{\prime}$ significantly up-weights the importance of eigen components with large eigenvalues. 
Table 8: Regression of $\boldsymbol{v}_{(h)}^{\prime}$ on $\boldsymbol{v}^{\prime}$ for pre-WWII Germany and Japan

\begin{tabular}{cccccccccc}
\hline & \multicolumn{4}{c}{ Germany } & & \multicolumn{5}{c}{ Japan } \\
\cline { 2 - 7 } \cline { 7 - 9 }$h$ & 1 & 2 & 3 & 4 & & 1 & 2 & 3 & 4 \\
\hline slope & 0.87 & 0.95 & 1.02 & 10.4 & & 0.64 & 0.65 & 0.91 & 0.92 \\
$R^{2}$ & 0.67 & 0.66 & 0.85 & 0.87 & & 0.49 & 0.65 & 0.92 & 0.92 \\
\hline
\end{tabular}

Table 9 describes the first three eigenvectors for these economies by listing the 5 largest sectoral entries by absolute value for each eigenvector. For Germany, the first two eigenvectors are highly correlated and represent the iron, steel, vehicles and aerospace industries. The first eigenvector also loads strongly on the spirits industry but the loading is negated by the second eigenvector. The third eigenvector loads on textile, clothing, fuel, and nonferrous metals. For Japan, because the input-output table only contains 23 sectors, we have to examine the industry structures with limited resolution. Nevertheless, the first eigenvector loads strongly on metals, machinery, and construction; the first three eigenvectors also jointly loads on textiles, office supplies, printing and publishing, and leather and rubber products.

Proposition 2 characterizes the entire path of the sectoral output vector $\ln \boldsymbol{q}(t)$ as a function of the initial shock vector $\tilde{\boldsymbol{z}}$ and time. One can also apply the proposition to compute the half-lives of shocks, as summarized in the proposition below.

Proposition 6. The half-life $t_{1 / 2}^{i}$ of temporary TFP shock vector $-\tilde{\boldsymbol{z}}$ on sector $i$ 's output is the solution to

$$
\boldsymbol{e}_{i}^{\prime} \boldsymbol{\Sigma}(\boldsymbol{I}-\boldsymbol{\Sigma})^{-1}\left(\frac{1}{2} \boldsymbol{I}-e^{-\delta^{-1}(\boldsymbol{I}-\boldsymbol{\Sigma}) t_{1 / 2}^{c}}\right) \tilde{\boldsymbol{z}}=0 .
$$

The half-life $t_{1 / 2}^{c}$ of temporary TFP shock vector $-\tilde{\boldsymbol{z}}$ on aggregate consumption is the solution to

$$
\boldsymbol{\beta}^{\prime} \boldsymbol{\Sigma}(\boldsymbol{I}-\boldsymbol{\Sigma})^{-1}\left(\frac{1}{2} \boldsymbol{I}-e^{-\delta^{-1}(\boldsymbol{I}-\boldsymbol{\Sigma}) t_{1 / 2}^{c}}\right) \tilde{\boldsymbol{z}}=0 .
$$

where $\boldsymbol{e}_{i}$ is the elementary vector with $i$-th entry being 1 and all other entries zero.

Intuitively, a shock to sector $j$ has a long half-life on sector $i$ 's output if a significant fraction of $j$ 's direct-and-indirect sales to $i$ are generated through higher rounds of linkages.

We now separately shock the productivity of each sector $i=1, \ldots, N$, and we visualize the output response (relative to the initial steady-state) in every other sector $j=1, \ldots, N$, for the short run $(t=0)$ and the medium run $(0<t<\infty)$. We do not make precise how long does the "medium run" correspond to; the point we try to convey here is not quantitative but is instead the qualitative feature that shocks to certain sectors, such as metals, tend to having lasting impact in the economy. 


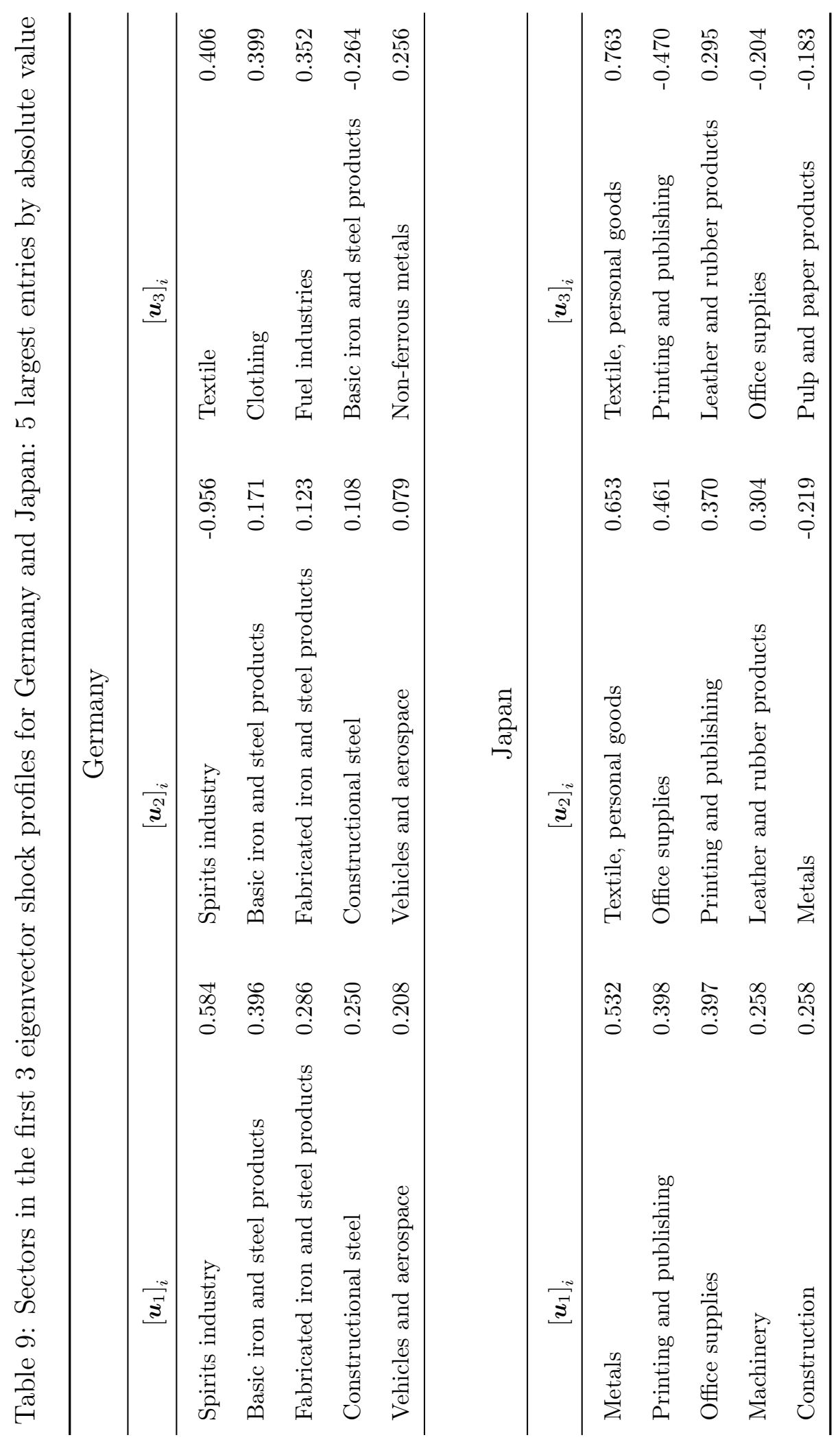


In Figure 7, we use chord diagrams to show cross-sector exposure to productivity shocks at different time scales for pre-WWII Germany. Sectors are arranged around a circle, where the size of the inner segment for each country shows its overall outward exposure (the effect of its productivity shocks on other sectors), and the gap between the inner and outer segments shows its overall inward exposure (the effect of other productivity shocks upon it). Arrows emerging from the inner segment for each sector show the cross-industry impact of its productivity shocks on output in other sectors. Arrows pointing towards the gap between the inner and outer segments show the impact of other sector's productivity growth on its output. For ease of visualization, only the largest $10 \%$ of cross exposures are shown at each time scale.

Panel (a) of Figure 7 shows the short run cross-industry exposures. Shocks to basic iron and steel products, transportation and communication, utilities such as electricity, gas and water, and other services tend to reduce the output in many other sectors. Panel (b) of Figure 7 shows the medium run cross-industry exposures. Note that the size of arrows are comparable within each panel but not across panels (a) and (b). Relative to panel (a), the most salient feature in panel (b) is the persistence and relative importance of shocks to basic iron and steel products: it's medium run impact overshadows shocks to any other sector of the economy.

Figure 7: Sectoral cross-exposure in pre-WWII Germany

(a) Short run

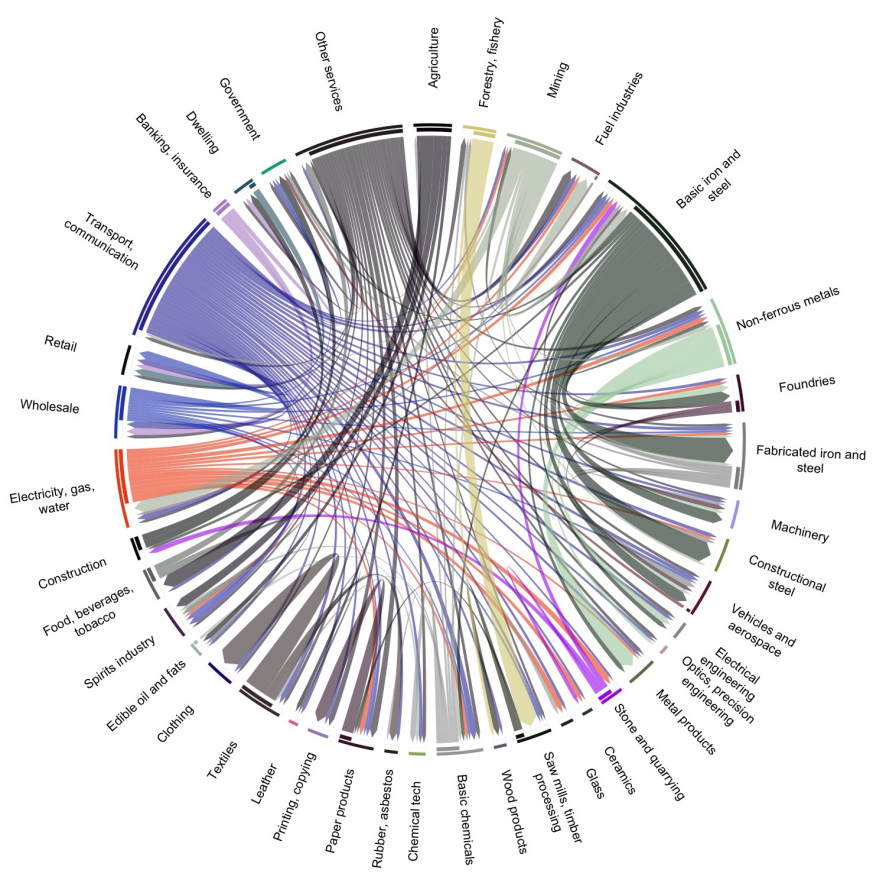

(b) Medium run

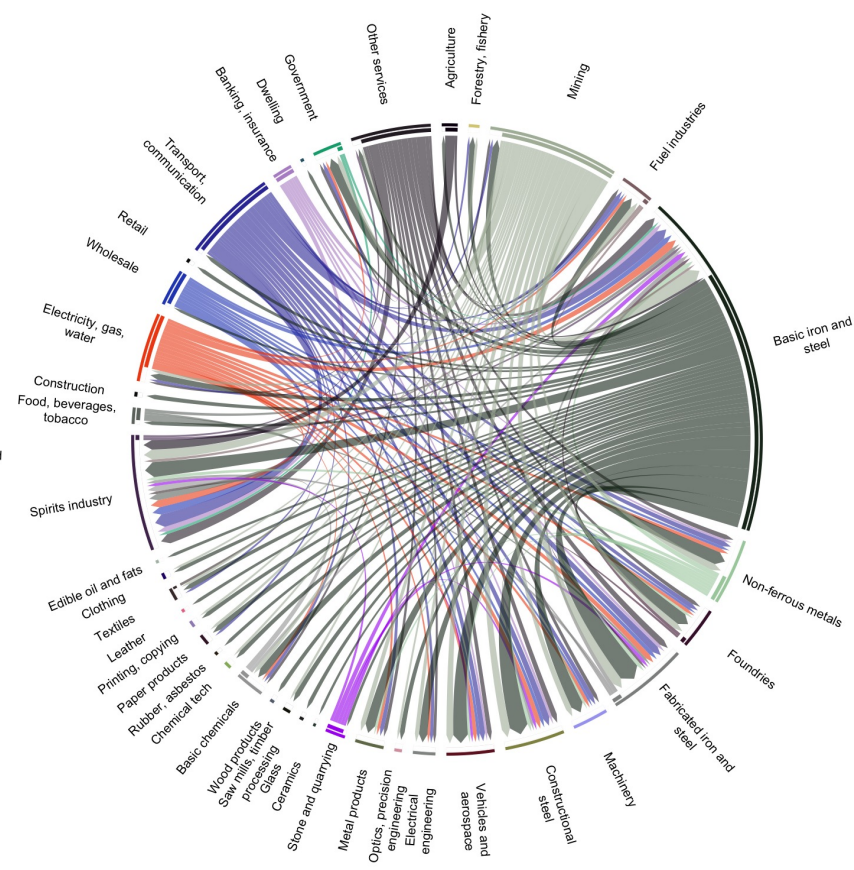


Figure 8: Sectoral cross-exposure in pre-WWII Japan

(a) Short run

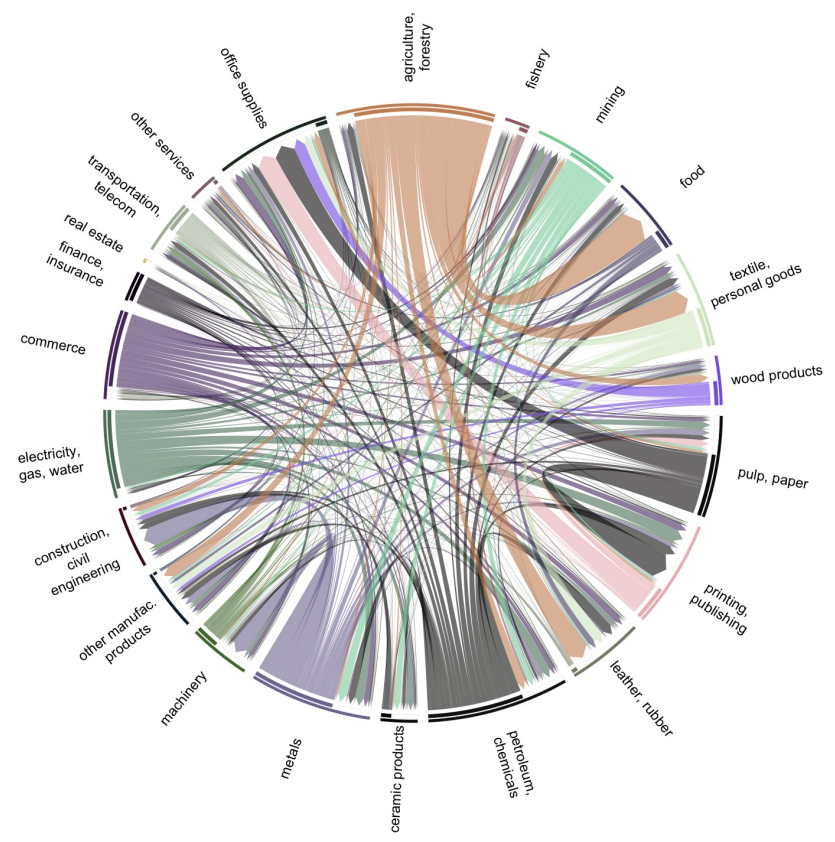

(b) Medium run

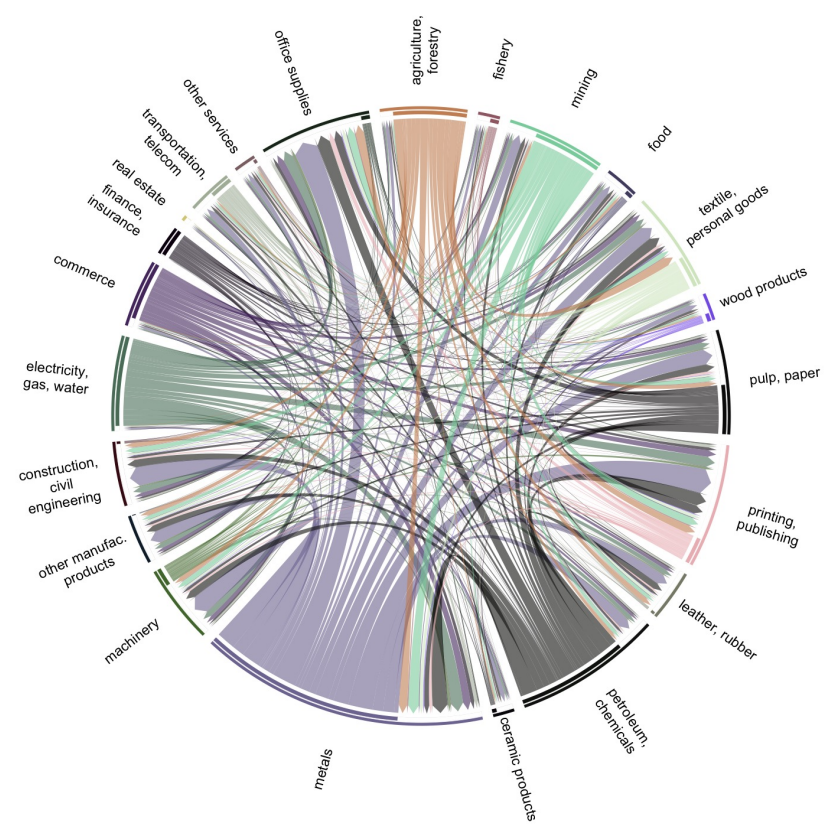

Figure 8 illustrates the short- and medium-run cross-sector exposures for pre-WWII Japan. Once again, comparing panels (a) and (b), the most salient feature is that shocks to metals tend to have long lasting impact on many other sectors of the economy. In addition, shocks to petroleum and chemicals, as well as to the electricity, gas, and water sector also gain relative importance over time.

In Figures 9 and 10, we use heat-maps to show each sector's output exposure to temporary TFP shocks to every other sector at different time scales for the pre-WWII Germany and Japan, respectively. The columns represent sectors being shocked, and the rows represent exposed sectors. Darker shades of red indicate more negative output impact at a given time. Similar to the chord diagrams in Figure 7, the heat-map of Figure 9 indicates that shocks to iron and steel products, non-ferrous metals, transportation, and communication sectors have long lasting impact to outputs of many other sectors in the pre-war Germany, as evident from the darker reds for the corresponding columns. Likewise, Figure 10 shows that shocks to metals, petroleum, chemicals, and electricity, gas, and water sectors have long lasting impact to many other sectors. 
Figure 9: Sectoral cross-exposure over time in pre-WWII Germany

(a) Initial impact, $t=0$

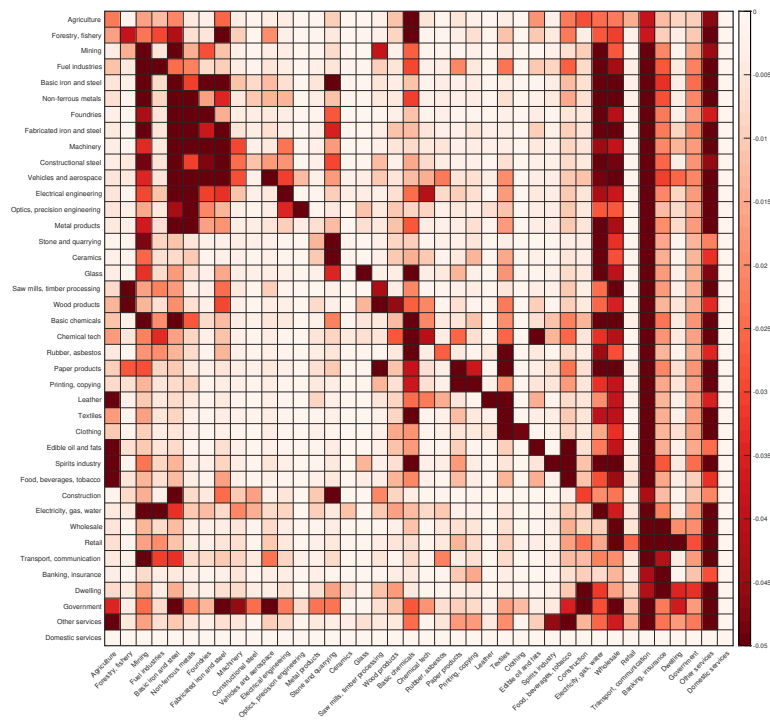

(c) After some more time...

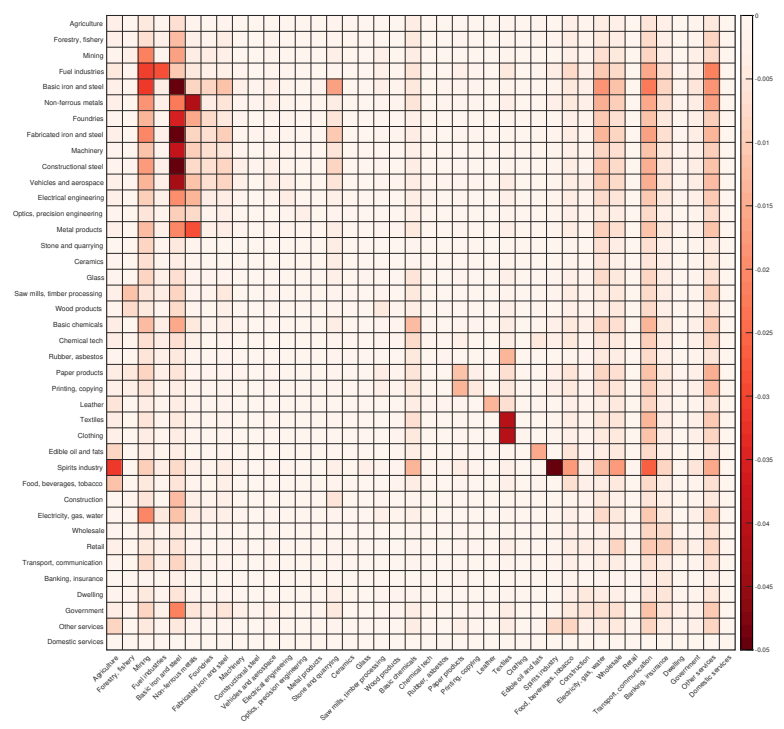

(b) After some time...

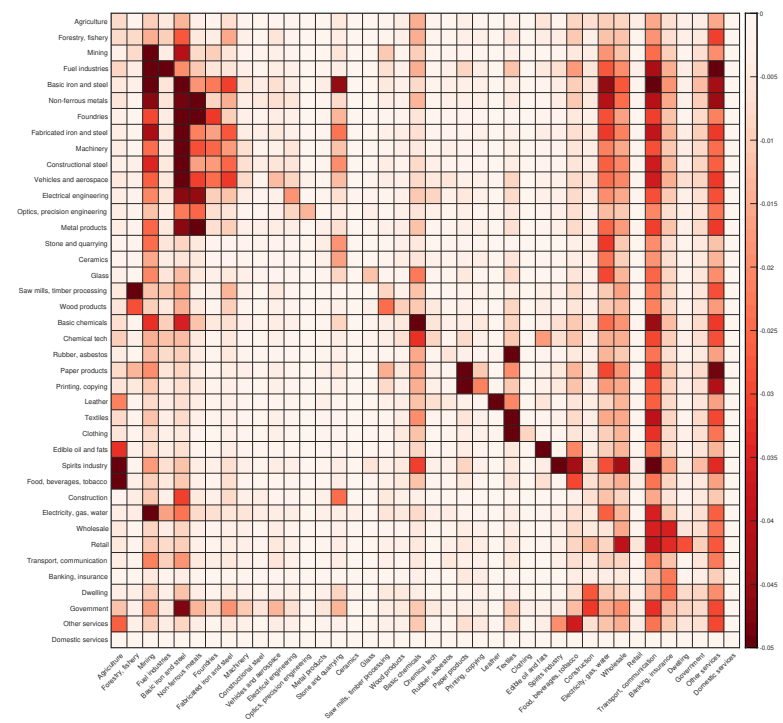

(d) After a long time...

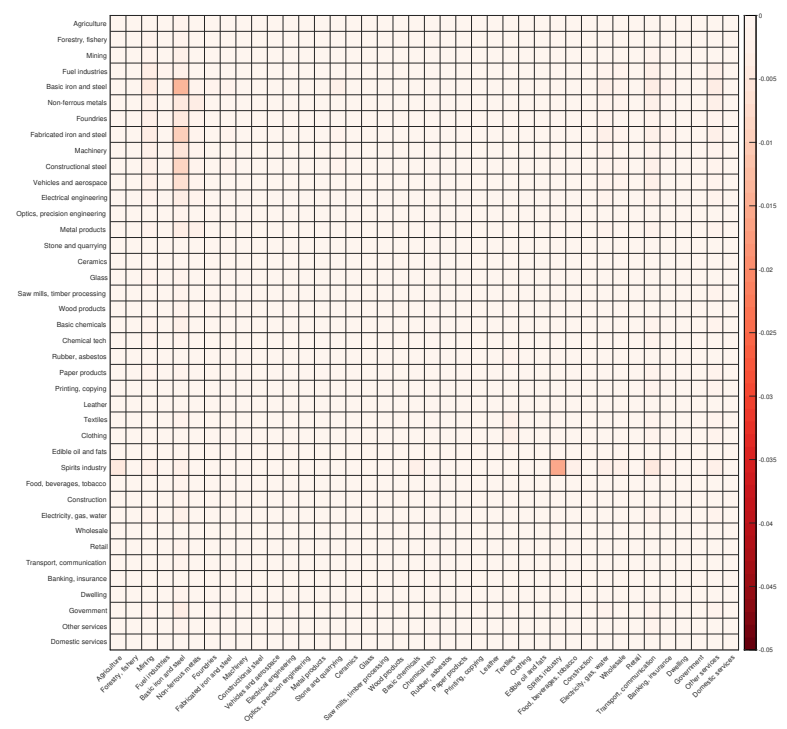


Figure 10: Sectoral cross-exposure over time in pre-WWII Japan

(a) Initial impact, $t=0$

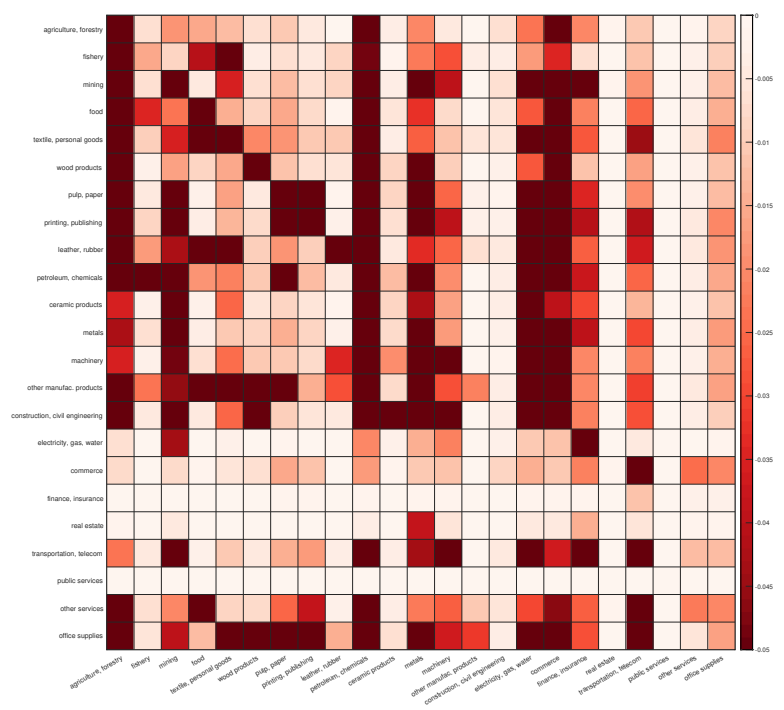

(c) After some more time...

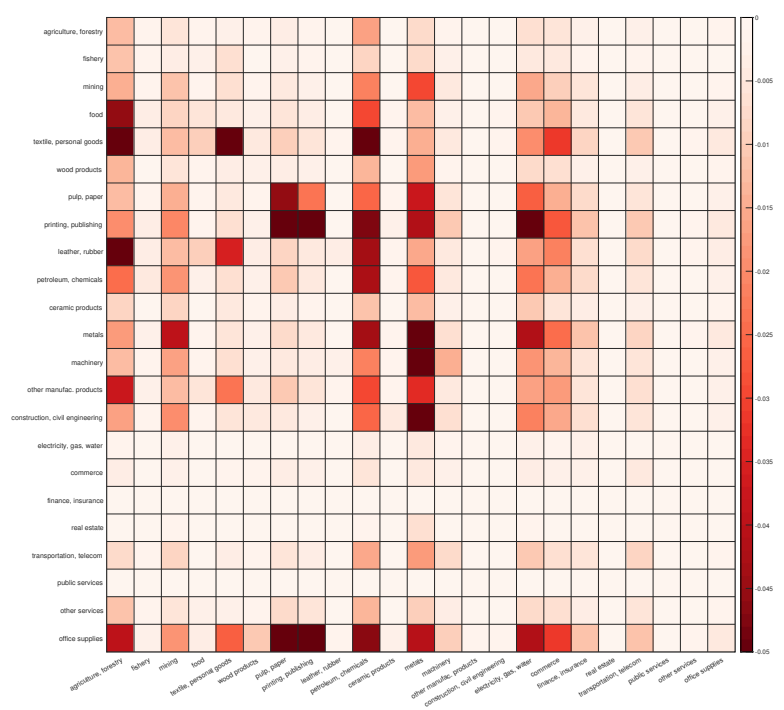

(b) After some time...

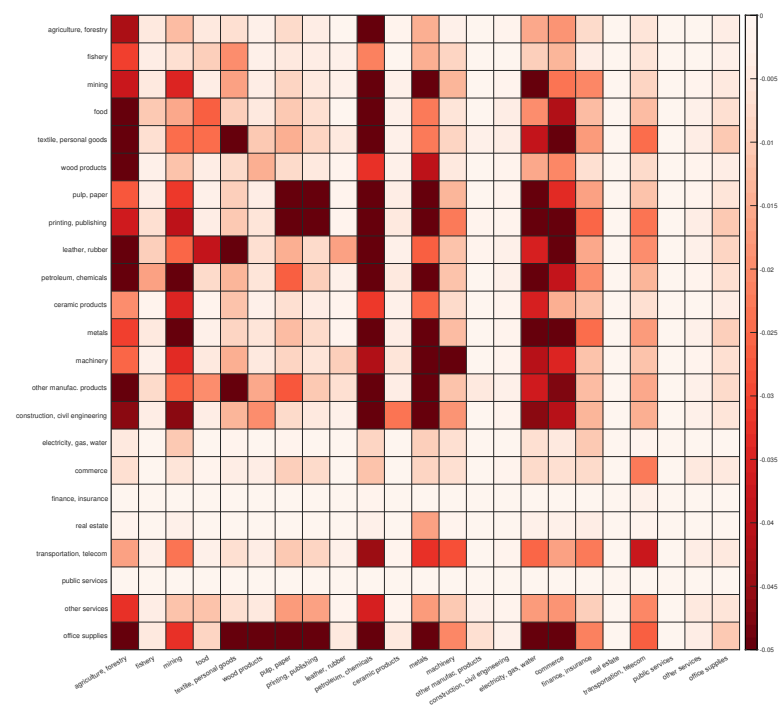

(d) After a long time...

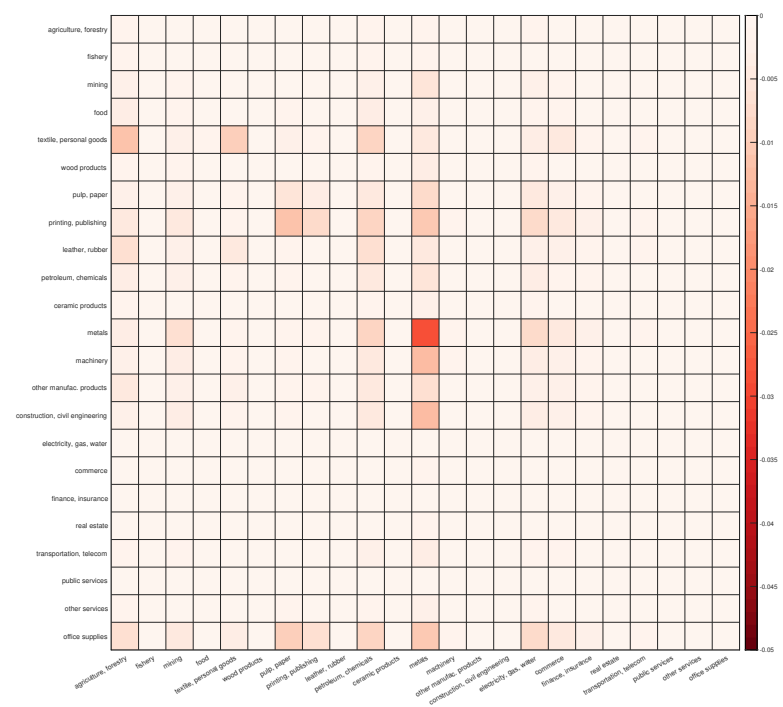

\section{Conclusion}

The dynamical view of the input-output matrix that is inherent in spectral graph theory and dynamical system theory can reveal important determinants of the structure of production networks. We have built one such dynamical system - a microfounded general equilibrium model with adjustment frictions in which economy gradually transition to the steady state. 
This analysis is useful to reveal the structure of the input-output matrix - varying the degree of the frictions and thus the speed of the adjustment allows us to have a multi-scale representation of the economy. These scales represent the varying importance of the higher order links and the associated eigenvalues and eigenvectors. We apply our analysis to the production networks of the modern day US, and those of the WWII era Nazi Germany and Imperial Japan.

\section{References}

Acemoglu, D., U. Akcigit, And W. Kerr (2015): "Networks and the Macroeconomy: An Empirical Exploration," 273-335.

Acemoglu, D., V. M. Carvalho, A. Ozdaglar, and A. Tahbaz-Salehi (2012): "The Network Origins of Aggregate Fluctuations," Econometrica, 80, 1977-2016.

Adena, M., R. Enikolopov, M. Petrova, and H. J. Voth (2020): "Bombs, Broadcasts and Resistance: Allied Intervention and Domestic Opposition to the Nazi Regime During World War II," .

Antràs, P., D. Chor, T. Fally, And R. Hillberry (2012): "Measuring the Upstreamness of Production and Trade Flows," American Economic Review, 102, 412-16.

BaqAeE, D. R. (2018): "Cascading Failures in Production Networks," Econometrica, 86.

BaqAeE, D. R. And E. FARHi (2019): "The Macroeconomic Impact of Microeconomic Shocks: Beyond Hulten's Theorem," Econometrica, 87.

- (2020): "Productivity and Misallocation in General Equilibrium," The Quarterly Journal of Economics, 135, 105-163.

Benzi, M. And C. Klymko (2015): "On the Limiting Behavior of Parameter-Dependent Network Centrality Measures," SIAM Journal on Matrix Analysis and Applications, 36, 686-706.

Berman, A. And R. J. Plemmons (1994): Nonnegative Matrices in the Mathematical Sciences, Society for Industrial and Applied Mathematics.

Bigio, S. And J. LA'O (2020): "Distortions in Production Networks," The Quarterly Journal of Economics, 135, 2187-2253.

Bloch, F., M. O. Jackson, and P. Tebaldi (2020): "Centrality Measures in Networks," Working paper. 
Bollard, A. (2020): Economists at War: How a Handful of Economists Helped Win and Lose the World Wars, Oxford University Press.

Carvalho, V. M. (2014): "From Micro to Macro via Production Networks," Journal of Economic Perspectives, 28, 23-48.

Carvalho, V. M., M. Nirei, Y. U. Saito, and A. Tahbaz-Salehi (forthcoming): "Supply Chain Disruptions: Evidence from the Great East Japan Earthquake," Quarterly Journal of Economics.

Carvalho, V. M. and A. Tahbaz-Salehi (2019): "Production Networks: A Primer," Annual Review of Economics, 11, 635-663.

Chung, F. R. K. (1997): Spectral Graph Theory, vol. 92 of CBMS Regional Conference Series in Mathematics, American Mathematical Society.

Coifman, R. R., I. G. Kevrekidis, S. Lafon, M. Maggioni, and B. Nadler (2008): "Diffusion maps, reduction coordinates, and low dimensional representation of stochastic systems," Multiscale Modeling \& Simulation, 7, 842-864.

Colonius, F. And W. Kliemann (2014): Dynamical Systems and Linear Algebra, American Mathematical Society.

Davis, D. R. And D. E. Weinstein (2002): "Bones, Bombs, and Break Points: The Geography of Economic Activity," American Economic Review, 92, 1269-1289.

Fremdling, R. And R. Staeglin (01 Nov. 2014): "An Input-Output Table for Germany in 1936: A Documentation of Results, Sources and Research Strategy," Jahrbuch f $\widetilde{A} E r$ Wirtschaftsgeschichte / Economic History Yearbook, 55, 187 - 298.

Golub, B., M. Elliot, And M. V. Leduc (2020): "Supply Network Formation and Fragility," Working paper.

Grassi, B. And J. Sauvagnat (2019): "Production networks and economic policy," Oxford Review of Economic Policy, 35, 638-677.

GRIGOR'YAN, A. (2018): Introduction to analysis on graphs, vol. 71, American Mathematical Society.

Guglielmo, M. (2008): "The Contribution of Economists to Military Intelligence during World War II," The Journal of Economic History, 68, 109-150.

HARrison, M. (2020): "Economic warfare: Insights from Mançur Olson," The Economics of the Second World War: Eighty Years On (VoxEU). 
Hulten, C. R. (1978): "Growth Accounting with Intermediate Inputs," The Review of Economic Studies, 45, 511-518.

Jackson, M. O., B. Rogers, And Y. Zenou (forthcoming): "Networks: An Economic Perspective," Oxford Handbook of Social Network Analysis.

Jones, C. I. (2011): "Intermediate Goods and Weak Links in the Theory of Economic Development," American Economic Journal: Macroeconomics, 3, 1-28.

- (2013): "Misallocation, Economic Growth, and Input-Output Economics," Advances in Economics and Econometrics, 2.

KATZ, L. (1953): "A New Status Index Derived from Sociometric Analysis," Psychometrika.

Kemeny, J. G. And J. L. Snell (1960): Finite Markov Chains, Van Nostrand, Princeton.

Liu, E. (2019): "Industrial Policies in Production Networks*," The Quarterly Journal of Economics, 134, 1883-1948.

Nishikawa, S. AND H. Koshinara (1981): "Input-Output Table of 1935," Interwar Japanese Economy (ed. by Takafusa Nakamura).

OBerfield, E. (2018): "A Theory of Input-Output Architecture," Econometrica, 86.

Olson, M. (1962): "The Economics of Target Selection for the Combined Bomber Offensive," Royal United Services Institution. Journal, 107, 308-314.

Rostow, W. W. (1981): Pre-Invasion Bombing Strategy, University of Texas Press.

SAlant, W. (1942): "The Selection of Industrial Bombing Targets: Some Analytical Notes," Memorandum to EOU, in Rostow (1981), "Pre-Invasion Bombing Strategy".

Spielman, D. (2019): Spectral and Algebraic Graph Theory.

Steinerberger, S. And A. Tsyvinski (2019): "Tax Mechanisms and Gradient Flows," .

The E.O.U. Special Report No.9 (1943): "Timing of Consequences of A Bombing Program," in Rostow (1981), "Pre-Invasion Bombing Strategy". 


\section{Appendix}

\section{A Factor Structure of the U.S. Input-Output Table: Al- ternative Values of $\rho$ and $\delta$}

In Section 5, we demonstrate the factor structure of the U.S. input-output table under the assumption that $\rho=\delta=10 \%$. This is because the importance of the $k$-th eigen component in explaining $\boldsymbol{v}^{\prime}$ is $\left|\boldsymbol{\beta}^{\prime} \boldsymbol{u}_{k} \frac{\lambda_{k}}{\left(1-\lambda_{k}\right)\left(1+\rho \delta-\lambda_{k}\right)}\right|$, which decays rapidly as $\lambda_{k}$ decreases. In this Appendix, we replicate Figure 3 panel (f), Table 2, and Figure 5 to demonstrate that the factor structure is robust to alternative values of $\rho$ and $\delta$. In fact, the factor structure is robust even if $\rho \delta \rightarrow \infty$, where the relative importance of the $k$-th eigenvector decays the slowest in $\left|\lambda_{k}\right| .^{8}$

Figure 11: Replication of Figure 3 panel (f) under alternative values of $\rho$ and $\delta$ : decay of eigen components
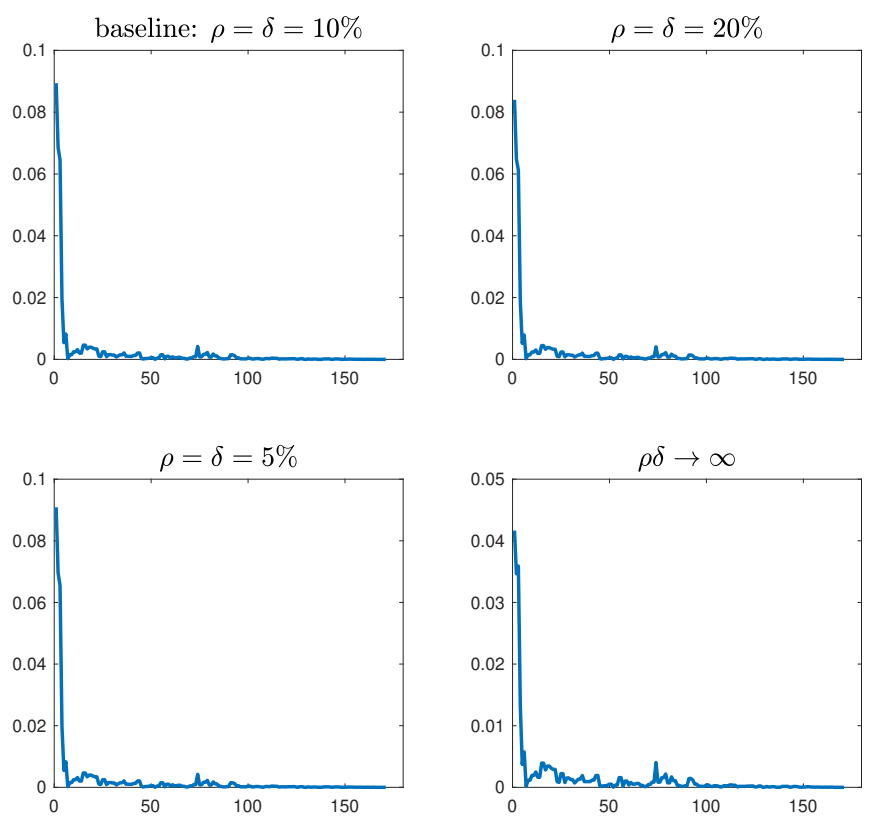

\footnotetext{
${ }^{8}$ That is, for $k \geq 1,\left|\lambda_{k}\right| \leq\left|\lambda_{1}\right|$, and $\left|\boldsymbol{\beta}^{\prime} \boldsymbol{u}_{k} \frac{\lambda_{k}}{\left(1-\lambda_{k}\right)\left(1+\rho \delta-\lambda_{k}\right)}\right| /\left|\boldsymbol{\beta}^{\prime} \boldsymbol{u}_{1} \frac{\lambda_{1}}{\left(1-\lambda_{1}\right)\left(1+\rho \delta-\lambda_{1}\right)}\right|$ is increasing in $\rho \delta$ and converges to $\left|\boldsymbol{\beta}^{\prime} \boldsymbol{u}_{k} \frac{\lambda_{k}}{1-\lambda_{k}}\right| /\left|\boldsymbol{\beta}^{\prime} \boldsymbol{u}_{1} \frac{\lambda_{1}}{1-\lambda_{1}}\right|$ as $\rho \delta \rightarrow \infty$.
} 
Table 10: Replication of Table 2 as $\rho \delta \rightarrow \infty$ : regression of $\boldsymbol{v}_{(h)}^{\prime}$ on $\boldsymbol{v}^{\prime}$

\begin{tabular}{ccccccc}
\hline$h$ & 1 & 2 & 3 & 4 & 5 & 6 \\
\hline slope & 0.37 & 0.50 & 0.87 & 0.90 & 0.91 & 0.90 \\
\hline$R^{2}$ & 0.24 & 0.24 & 0.59 & 0.90 & 0.89 & 0.85 \\
\hline
\end{tabular}

Figure 12: Replication of Figure 5 as $\rho \delta \rightarrow \infty$ : welfare impact from the initial eigenvectors $\left(\boldsymbol{v}_{(h)}^{\prime}\right)$ plotted against $\boldsymbol{v}^{\prime}$

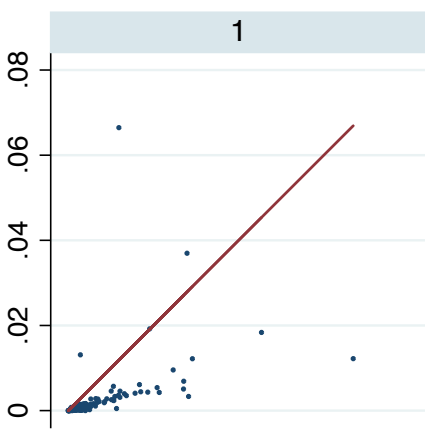

4

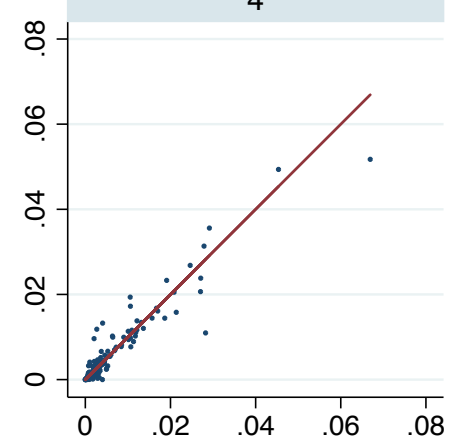

2

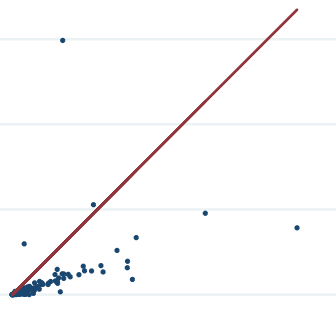

5

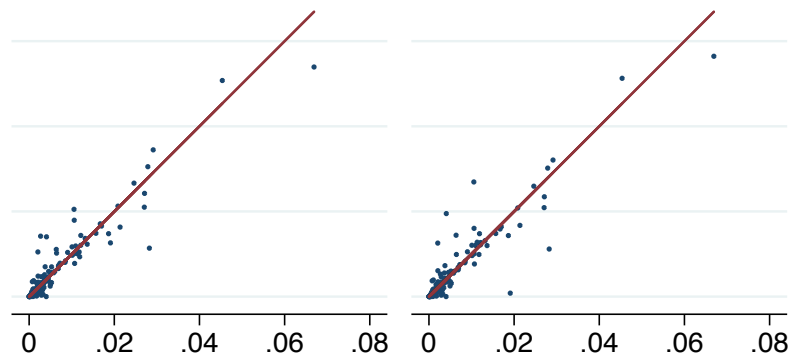





\section{B The Input-Output of Japan in 1935}

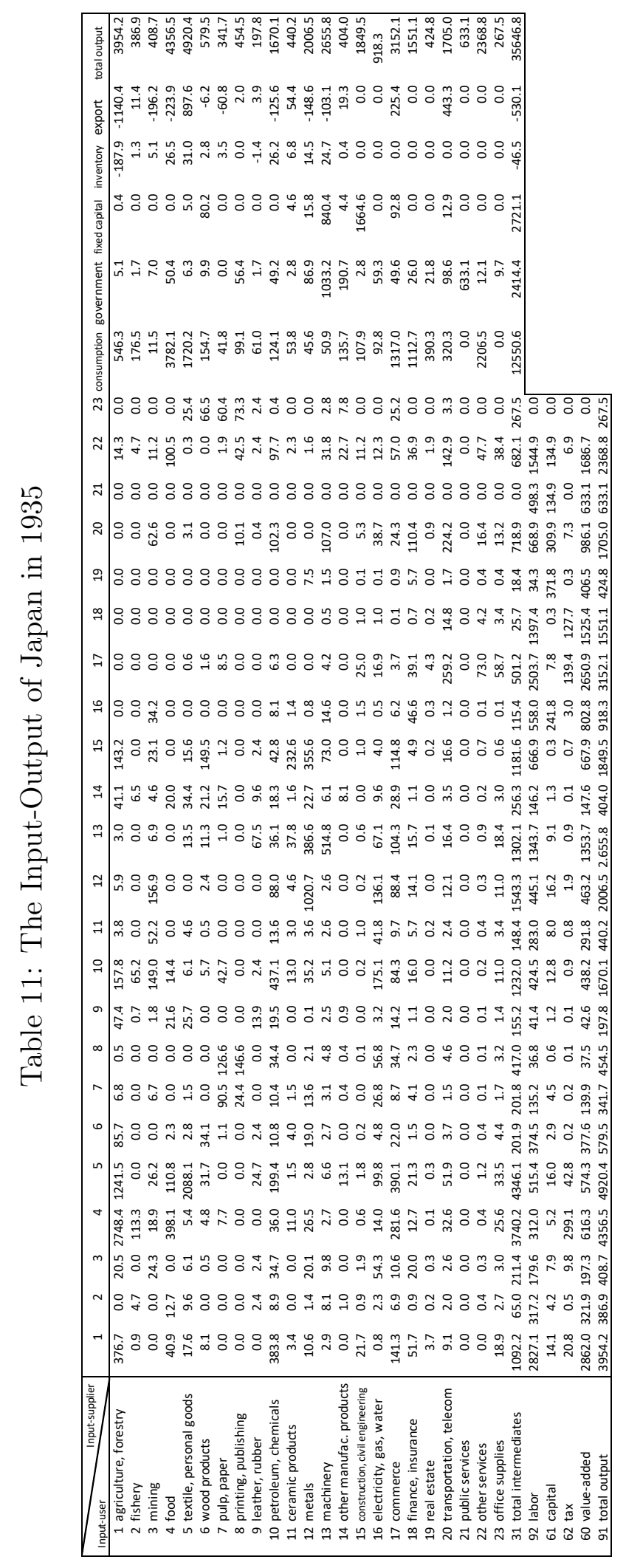

